\title{
Collinearity assessment of geocentre coordinates derived from multi-satellite SLR data
}

\author{
Ciprian B. Spatar · Philip Moore · Peter J. Clarke \\ This is an author preprint of an article published in the Journal of Geodesy. The final \\ publication is available at Springer via http://dx.doi.org/10.1007/s00190-015-0845-x
}

Accepted by the Journal of Geodesy on 27 July 2015

\begin{abstract}
Of the three satellite geodetic techniques contributing to the International Terrestrial Reference Frame (ITRF), Satellite Laser Ranging (SLR) is generally held to provide the most reliable time series of geocentre coordinates and exclusively defines the ITRF origin. Traditionally, only observations to the two LAser GEOdynamics Satellite (LAGEOS) and Etalon pairs of satellites have been used for the definition of the ITRF origin. Previous simulation studies using evenly sampled LAGEOS-like data have shown that only the $Z$ component of geocentre motion suffers minor collinearity issues, which may explain its lower quality compared to the equatorial components. Using collinearity diagnosis, this study provides insight into the actual capability of SLR to sense geocentre motion using the existing geographically unbalanced ground network and real observations to eight spherical geodetic satellites. We find that, under certain parameterisations, observations to the low Earth orbiters (LEOs) Starlette, Stella, Ajisai and LAser RElativity Satellite (LARES) are able to improve the observability of the geocentre coordinates in multi-satellite solutions compared to LAGEOS-only solutions. The higher sensitivity of LEOs to geocentre motion and the larger number of observations are primarily responsible for the improved observability. Errors in the modelling of Starlette, Stella and Ajisai orbits may contaminate the geocentre motion estimates, but do not disprove the intrinsic strength of LEO tracking data. The sporadically observed Etalon satellites fail to make a significant beneficial contribution to the observability of the geocentre coordinates derived via the network shift approach and can be safely omitted from SLR
\end{abstract}

\footnotetext{
C. B. Spatar · P. Moore · P. J. Clarke

School of Civil Engineering and Geosciences, Newcastle University,

Newcastle upon Tyne, NE1 7RU, UK

E-mail: c.b.spatar@ncl.ac.uk
}

data analyses for terrestrial reference frame (TRF) determination.

Keywords SLR · Geocentre · Collinearity · Observability · Orbit determination - Terrestrial reference frame .

LAGEOS $\cdot$ Etalon $\cdot$ Starlette $\cdot$ Stella $\cdot$ Ajisai $\cdot$ LARES

\section{Introduction}

Geocentre motion has attracted increasing interest in recent years due to its relevance for TRFs (Lavallée et al 2006; Collilieux et al 2009; Wu et al 2011; Dong et al 2014), ocean and ice mass balance estimates (Chambers et al 2004; Chambers 2006; Barletta et al 2013), geocentric sea level observations (Chen et al 2005; Collilieux and Wöppelmann 2011; Baur et al 2013; Melachroinos et al 2013) and other geodetic products (Wu et al 2012). Geocentre variations are induced by the redistribution of fluid mass within and at the surface of the Earth at time scales ranging from sub-daily to secular. The differential motion of the centre of mass of the entire Earth system (i.e. the solid Earth and its fluid envelope), denoted as $\mathrm{CM}$, with respect to the centre of surface figure $(\mathrm{CF})$ is conventionally adopted as the definition of geocentre motion (Petit and Luzum 2010). The CF frame is theoretically realised by a uniform and infinitely dense network of ground stations. Its ideal nature prompted $\mathrm{Wu}$ et al $(2002,2003)$ to propose the centre of network (CN) frame, the origin of which is the centre of the polyhedron formed by all operating stations at a particular epoch.

Since artificial satellites orbit about the instantaneous CM, all satellite geodetic techniques should theoretically be capable of sensing geocentre motion. However, due to technique-specific errors, the quality of GNSS and Doppler Orbitography and Radiopositioning Integrated by Satellite (DORIS) geocentre motion estimates is restricted. For both techniques, the complex satellite geometries complicate 
the modelling of solar radiation pressure (SRP) effects. Mismodelling of SRP introduces spurious signals in the time series of geocentre coordinates estimated from GNSS (Meindl et al 2013) and DORIS data (Gobinddass et al 2009a), particularly along the $Z$ axis. GNSS geocentre motion estimates are particularly vulnerable to modelling errors due to their strong collinearity with other parameters routinely estimated in standard GNSS data analyses, as demonstrated by Rebischung et al (2014). The agreement between DORIS- and SLR-derived geocentre coordinates can be improved by empirically rescaling the SRP model of each satellite using time-dependent coefficients (Gobinddass et al 2009b).

Laser ranges to passive geodetic satellites are among the most unambiguous and accurate observation types in satellite geodesy. The zenith atmospheric delays of laser signals can be accurately determined (Mendes and Pavlis 2004) and mapped for elevation angles above $3^{\circ}$ (Mendes et al 2002), without the necessity to estimate any tropospheric parameters. Moreover, the spherical shapes and favourable areato-mass ratios of geodetic satellites facilitate the modelling of non-gravitational forces acting on their surfaces. These are among the reasons why the origin of the International Terrestrial Reference Frame 2008 (ITRF2008), the latest realisation of the International Terrestrial Reference System (ITRS), is defined solely by SLR measurements (Altamimi et al 2011). The ITRF origin resembles a mean CM at secular time scales and $\mathrm{CF}$ (or $\mathrm{CN}$ ) at seasonal and shorter time scales (Dong et al 2003). Its current stability was appraised to be around $0.5 \mathrm{~mm} \mathrm{yr}^{-1}$ for the equatorial components but worse by a factor of two along the axial component (Wu et al 2011; Argus 2012), lower than the standard of $0.1 \mathrm{~mm} \mathrm{yr}^{-1}$ set by the Global Geodetic Observing System (GGOS) for geosciences applications (Gross et al 2009). Considering the slow development and densification of the SLR network, accuracy improvements of the ITRF origin in the near future will most likely arise from advances in SLR data modelling.

Traditionally, the LAGEOS- 1 and 2 and Etalon- 1 and 2 medium Earth orbiters (MEOs) have been used for TRF determination with SLR. The combined solution submitted by the International Laser Ranging Service (ILRS) for the computation of ITRF2008 resulted from the analysis of LAGEOS-1 data spanning the period 1983.0-1992.8, LAGEOS- 1 and 2 data from 1992.8 to 2002.5 and a combination of LAGEOS- 1 and 2 and Etalon- 1 and 2 observations over the period 2002.5-2009.0. ${ }^{1}$ The LEOs Starlette, Stella and Ajisai have been predominantly used, in conjunction with LAGEOS-1 and 2, for recovering low degree and order (d/o) geopotential coefficients (Cheng et al 1997; Cheng and Tapley 1999; Moore et al 2005; Maier et al 2012). Recent studies (Cheng et al 2013; Sośnica et al 2014) reported geo-

\footnotetext{
1 http://ilrs.gsfc.nasa.gov/docs/ILRS_contribution_to_ ITRF2008.pdf
}

centre coordinates from combined LAGEOS-LEO data, but no conclusive evidence that multi-satellite solutions improve the observability of the geocentre coordinates compared to LAGEOS-1 and 2 solutions has been provided. The higher sensitivity of LEOs to the long-wavelength harmonics of the gravity field and the quasi-polar orbit of Stella are often speculatively quoted as arguments for the potentially superior quality of geocentre motion time series derived from multi-satellite data (Angermann and Müller 2008; Sośnica et al 2014). LARES data analysis results have yet to be widely reported due to the short tracking history of the satellite launched on February 13, 2012, but its inclusion in ILRS products is envisaged for the near future. ${ }^{2}$

Using simulated observations sampled every 5 minutes to two LAGEOS-like satellites and an ideal network of 100 stations, Rebischung et al (2014) concluded that the SLR geocentre determination is free of major collinearity issues, especially for the equatorial geocentre coordinates. In this study, we extend the analysis to sparse and asymmetric real networks acquiring measurements at irregular time intervals to provide an indication of the current capability of SLR to sense geocentre motion. Additionally, the potential of Etalon and LEO observations to strengthen the tie of the SLRderived TRF origin to CM, and thus improve the accuracy of the ITRF origin, is also assessed. Various satellite combinations and solution parameterisations are investigated. The conclusions of this study apply exclusively to the network shift approach, a translational procedure for the determination of geocentre motion that has been in extensive use over the past two decades (Bouillé et al 2000; Crétaux et al 2002; Moore and Wang 2003; Feissel-Vernier et al 2006; Collilieux et al 2009).

Section 2 introduces the concept of collinearity in geodetic parameter estimation by least squares and outlines the generalised technique proposed by Rebischung et al (2014) for detecting collinearity problems among geodetic parameters, with particular emphasis on geocentre coordinates. The satellite data and the standards and conventions adopted for SLR data processing are described in Sect. 3. Section 4 presents the results obtained following the application of the collinearity diagnostic procedure to the determination of geocentre coordinates from multi-satellite SLR data via the network shift approach. Finally, Sect. 5 draws the conclusions of the study.

\section{Collinearity diagnosis in geodetic data analysis}

The problem of perfect collinearity among the parameters of a least squares problem arises when linear dependencies exist between the parameters. This issue translates to a rank

\footnotetext{
2 http://ilrs.gsfc.nasa.gov/docs/2014/Minutes_ILRS_ AWG_19th_ILW_2014.10.26_Meeting.pdf
} 
deficient design matrix A (i.e. at least one linear function of its columns equals the zero vector) and a singular normal matrix $\mathbf{N}$, a ubiquitous occurrence in geodetic analyses in the absence of a priori information. When $\mathbf{N}$ is near to singularity rather than singular, a unique but very unstable solution of the normal equations system can be determined. This latter complication is known as the collinearity (or multicollinearity) problem. In its presence, even minor changes in the data or in the functional model (e.g. the addition or removal of parameters) severely affect parameters suffering collinearity issues. Nearly collinear parameters are poorly determined from observational data and have large associated variances.

Collinearity can have dramatic effects on parameter estimation (Rawlings et al 1998; Montgomery et al 2012). Analysis of correlations between parameters can uncover pairwise near-linear dependencies, but it is unsuitable and inefficient for identifying severe non-orthogonality among several parameters. Proper detection and handling of collinearity problems are crucial for the estimation of geophysically meaningful and unbiased geodetic parameters. This section summarises the procedure developed by Rebischung et al (2014) for diagnosing the collinearity of geodetic parameters in general and, in particular, the collinearity of geocentre coordinates obtained through the network shift approach.

To reduce the rank defect $r$ of the design matrix $\mathbf{A}$, it is customary in geodesy to augment the normal equations with minimum (or inner) constraints (Blaha 1982), leading to the following system of conditional equations:

$$
\left[\begin{array}{cc}
\mathbf{N} & \mathbf{C} \\
\mathbf{C}^{\mathrm{T}} & \mathbf{0}
\end{array}\right]\left[\begin{array}{l}
\mathbf{x} \\
\mathbf{k}
\end{array}\right]=\left[\begin{array}{l}
\mathbf{b} \\
\mathbf{0}
\end{array}\right],
$$

where $\mathbf{C}$ is a $u \times r$ constraint matrix, $\mathbf{x}$ a vector of $u$ unknown parameters, $\mathbf{k}$ a vector of $r$ Lagrange multipliers and b the right-hand side of the normal equations system. The constraint matrix for station coordinates has a standard form (e.g Rebischung et al 2014). It removes the three orientation singularities by imposing a no-net-rotation condition with respect to the a priori reference frame and a fourth singularity stemming from the correlation between UT1-UTC and the longitudes of the orbital ascending nodes by fixing UT1UTC to a known value.

The network shift approach for geocentre motion determination involves the computation of six- or sevenparameter similarity transformations between successive CM-centred epoch reference frames and a secular frame such as ITRF2008. The average global displacement of the entire network forming an epoch reference frame is equated to the translation vector between the origins of the epoch frame and the secular frame. Geocentre information is inherently contained in the station coordinates estimated in the $\mathrm{CM}$ frame. The geocentre coordinates are therefore implicit parameters realised via station coordinates. Simultaneous estimation of geocentre coordinates and station coordinates may cause collinearity problems since the two interdependent parameter types represent similar information in different forms.

The signature (or effect of a unit variation) of an implicit parameter $y$ on the observations vector $\mathbf{I}$ can be expressed as

$\mathbf{s}_{y}=\frac{\partial \mathbf{l}}{\partial y}=\mathbf{A} \boldsymbol{\lambda}$

where $\boldsymbol{\lambda}=\partial \mathrm{x} / \partial y$ contains the partial derivatives of the explicit parameters $\mathbf{x}$ with respect to the implicit parameter $y$. The signatures of the three geocentre coordinates on stationsatellite ranges are as follows:

$$
\begin{aligned}
& \mathbf{s}_{X}=\mathbf{A}[\delta p, 0,0, \delta p, 0,0, \ldots, \delta p, 0,0,0,0,0,0, \ldots, 0]^{\mathrm{T}}, \\
& \mathbf{s}_{Y}=\mathbf{A}[0, \delta p, 0,0, \delta p, 0, \ldots, 0, \delta p, 0,0,0,0,0, \ldots, 0]^{\mathrm{T}}, \\
& \mathbf{s}_{Z}=\mathbf{A}[\underbrace{0,0, \delta p, 0,0, \delta p, \ldots, 0,0, \delta p}_{\text {station coordinates }}, \underbrace{0,0,0,0, \ldots, 0}_{\text {other parameters }}]^{\mathrm{T}},
\end{aligned}
$$

where $\delta p$ is the geocentre offset, assumed here to be identical along all three axes for simplicity. A positive geocentre offset along any axis has the effect of shortening the ranges to satellites orbiting in the positive hemisphere of the axis and lengthening the ranges to satellites in the negative hemisphere.

The first step for diagnosing the collinearity of a particular geocentre coordinate with the other parameters consists in introducing a fictitious geocentre offset (e.g. $\delta p=1 \mathrm{~cm}$ ) and calculating $\boldsymbol{\lambda}_{c}$ from the system

$$
\left[\begin{array}{cc}
\mathbf{N} & \mathbf{C} \\
\mathbf{C}^{\mathrm{T}} & \mathbf{0}
\end{array}\right]\left[\begin{array}{c}
\boldsymbol{\lambda}_{c} \\
\mathbf{k}
\end{array}\right]=\left[\begin{array}{c}
\mathbf{N} \boldsymbol{\lambda} \\
\mathbf{0}
\end{array}\right] .
$$

The partial derivatives $\boldsymbol{\lambda}_{c}$ represent the variations (under minimum constraints) of the explicit parameters due to a change in the implicit parameter.

In the second stage, the following system of equations is solved:

$$
\left[\begin{array}{ccc}
\mathbf{N} & \mathbf{C} & \boldsymbol{\lambda}_{c} \\
\mathbf{C}^{\mathrm{T}} & \mathbf{0} & \mathbf{0} \\
\boldsymbol{\lambda}_{c}^{\mathrm{T}} & \mathbf{0} & \mathbf{0}
\end{array}\right]\left[\begin{array}{c}
\mathbf{x}_{c} \\
\mathbf{k} \\
\mathbf{k}^{\prime}
\end{array}\right]=\left[\begin{array}{c}
\mathbf{0} \\
\mathbf{0} \\
\boldsymbol{\lambda}_{c}^{\mathrm{T}} \boldsymbol{\lambda}_{c}
\end{array}\right]
$$

Its solution is $\mathbf{x}_{c}=\boldsymbol{\lambda}_{c}-\boldsymbol{\gamma}_{y, c}$, where the term $-\boldsymbol{\gamma}_{y, c}$ represents the optimum response of the system to account for the artificially introduced offset $\boldsymbol{\lambda}_{c}$. Examination of $-\boldsymbol{\gamma}_{y, c}$ can reveal which explicit parameters are most affected by variations of the implicit parameter $y$ and thus responsible for its potential collinearity problems.

The final step of the collinearity diagnosis consists in computing the variance inflation factor (VIF) of $y$ using the 
formula

$V_{y, c}=\frac{\left\|\mathbf{s}_{y}\right\|^{2}}{\left\|\mathbf{s}_{y, c}^{p}\right\|^{2}}=\frac{\left\|\mathbf{A} \boldsymbol{\lambda}_{c}\right\|^{2}}{\left\|\mathbf{A} \mathbf{x}_{c}\right\|^{2}}$,

where $\mathbf{s}_{y, c}^{p}$ is the observable component of $\mathbf{s}_{y}$, known as the proper signature of $y$. If the full design matrix is unavailable, $\mathbf{A} \boldsymbol{\lambda}_{c}$ and $\mathbf{A} \mathbf{x}_{c}$ can be obtained by successively evaluating the functional model at the a priori parameters $\mathbf{x}_{0}, \mathbf{x}_{0}+\boldsymbol{\lambda}_{c}$ and $\mathbf{x}_{0}+\mathbf{x}_{c}$ and pairwise differencing the observation residuals.

The VIF is one of the most commonly used indicators of collinearity. It represents the ratio of the actual variance, $1 /\left\|s_{y, c}^{p}\right\|^{2}$, of a parameter and the theoretical variance, $1 /\left\|\mathbf{s}_{y}\right\|^{2}$, of the same parameter unaffected by collinearity problems. In an ideal case when the VIF equals unity, the investigated parameter is free of collinearity issues. A maximum value of 10 is generally considered admissible, but, as Rebischung et al (2014) suggested, this value is most likely unsuitable for geodetic problems involving hundreds to tens of thousands of parameters. Numerical VIF values vary logarithmically due to their quotient nature. To facilitate interpretation, each VIF value can be accompanied by a percentage value obtained from

$R_{y, c}=\sqrt{1-\frac{1}{V_{y, c}}}$,

where $R_{y, c}$ represents the multiple correlation coefficient of $y$ with the other parameters.

Collinearity diagnosis provides a platform for investigating the observability of parameters. The VIF, however, is defined as the ratio of two variances and thus lacks absolute meaning. Relative comparisons of VIFs are only meaningful when their values are derived from the same set of observations since the signature norm $\left\|\mathbf{s}_{y}\right\|$ and, implicitly, the variance $1 /\left\|\mathbf{s}_{y}\right\|^{2}$ of a parameter are constant when varying the analysis settings alone. The formal error $1 /\left\|s_{y, c}^{p}\right\|$ is generally a better measure of observability and has the advantage of being readily interpretable. Of particular interest for the observability of a parameter is how the formal error $1 /\left\|s_{y, c}^{p}\right\|$ compares to the required level of accuracy.

\section{Satellite data and processing strategy}

\subsection{Data, network and conventions}

Normal point (NP) observations to eight spherical geodetic satellites (i.e. LAGEOS-1 and 2, Etalon-1 and 2, Starlette, Stella, Ajisai and LARES) were homogeneously processed using an updated version of the precise orbit determination software Faust (Moore et al 1999). The data used in this study span 52 weeks from 30 December 2012 to 28 December 2013 and represent a combined data set retrieved from the two ILRS data centres, namely the Crustal

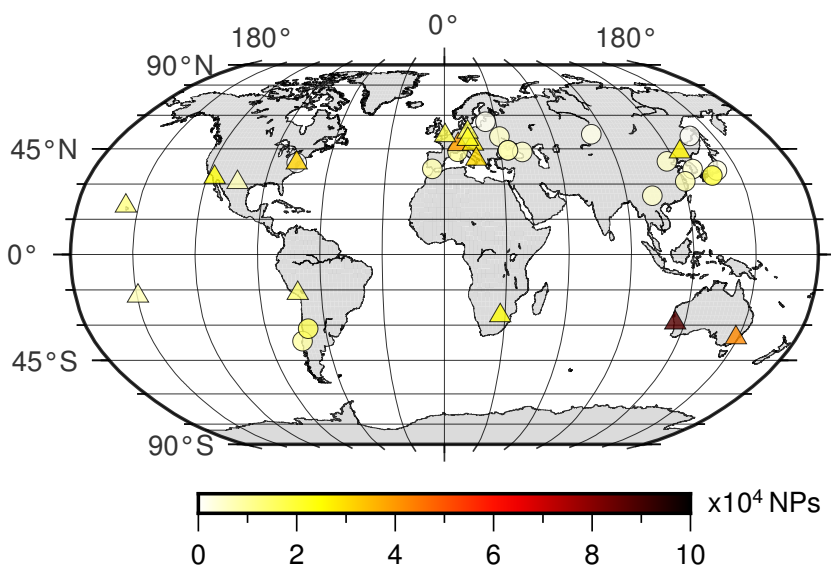

Fig. 1 The SLR network during the period 30 December 2012 - 28 December 2013. Core stations are represented by triangles and noncore stations by circles. The colour scale shows the total number of NPs to LAGEOS-1 and 2, Etalon-1 and 2, Starlette, Stella, Ajisai and LARES

Dynamics Data Information System (CDDIS; Noll 2010) and the EUROLAS Data Center (EDC). Satellite arcs, with a standard length of seven days, were simultaneously analysed in a multi-step iterative parameter estimation process. Four types of solutions involving different data combinations were generated in this study, namely (1) LAGEOS-1 and 2 solutions (which will be referred to as type A solutions), (2) LAGEOS-1 and 2 and Etalon-1 and 2 solutions (type B), (3) LAGEOS-1 and 2, Starlette, Stella and Ajisai solutions (type C), and (4) LAGEOS-1 and 2 and LARES solutions (type D).

A total of 34 SLR stations contribute to the analysed data set. Their spatial distribution and contributions to the data set are depicted in Fig. 1, which clearly illustrates the prevalence of stations located in the northern hemisphere of the Earth. The core network comprises 16 stations, 12 of which were chosen in accordance with ILRS recommendations for the time span of the data set. ${ }^{3}$ Monument Peak (7110), Tahiti (7124), Arequipa (7403) and Wettzell (8834) are the four additional stations part of the core network used in this study. The core set of stations serves as a reference network for the application of minimum constraints.

The SLR network is dynamic by nature due to several limiting factors. In contrast to GNSS, only one satellite can be observed by an SLR station at any moment of time. Furthermore, weather dependency prohibits the acquisition of regularly spaced observations over time. Finally, the complex equipment installed at SLR stations is susceptible to failures leading to interruptions in data collection and provision. An exemplification of the dynamic nature of the SLR network is given in Fig. 2, which depicts the percentages of stations situated in the positive $X, Y$ and $Z$ hemi-

\footnotetext{
3 http://ilrs.dgfi.badw.de/fileadmin/data_handling/
} ILRS_Dis continuities_File.snx 
Table 1 Selected orbital and physical parameters of the spherical geodetic satellites

\begin{tabular}{|c|c|c|c|c|c|c|c|}
\hline Satellite & $\begin{array}{l}\text { Perigee } \\
{[\mathrm{km}]}\end{array}$ & $\begin{array}{l}\text { Inclination } \\
{\left[^{\circ}\right]}\end{array}$ & $\begin{array}{l}\text { SRP } \\
\text { coefficient }\end{array}$ & $\begin{array}{l}\text { Diameter } \\
{[\mathrm{cm}]}\end{array}$ & $\begin{array}{l}\text { Mass } \\
{[\mathrm{kg}]}\end{array}$ & $\begin{array}{l}\text { Area-to-mass } \\
{\left[\mathrm{cm}^{2} \mathrm{~kg}^{-1}\right]}\end{array}$ & $\begin{array}{l}\text { Standard CoM } \\
\text { corr. }[\mathrm{mm}]\end{array}$ \\
\hline LAGEOS-1 & 5,860 & 109.8 & 1.130 & 60 & 406.965 & 6.948 & 251 \\
\hline LAGEOS-2 & 5,620 & 52.6 & 1.130 & 60 & 405.380 & 6.975 & 251 \\
\hline Etalon-1 & 19,120 & 64.9 & 1.240 & 129.4 & 1415 & 9.294 & 576 \\
\hline Etalon-2 & 19,120 & 65.5 & 1.280 & 129.4 & 1415 & 9.294 & 576 \\
\hline Starlette & 812 & 49.8 & 1.134 & 24 & 47.294 & 9.565 & 75 \\
\hline Stella & 800 & 98.6 & 1.131 & 24 & 48 & 9.425 & 75 \\
\hline Ajisai & 1,490 & 50.0 & 1.035 & 215 & 685.2 & 52.985 & 1,010 \\
\hline LARES & 1,450 & 69.5 & 1.125 & 36.4 & 386.8 & 2.690 & 133 \\
\hline
\end{tabular}

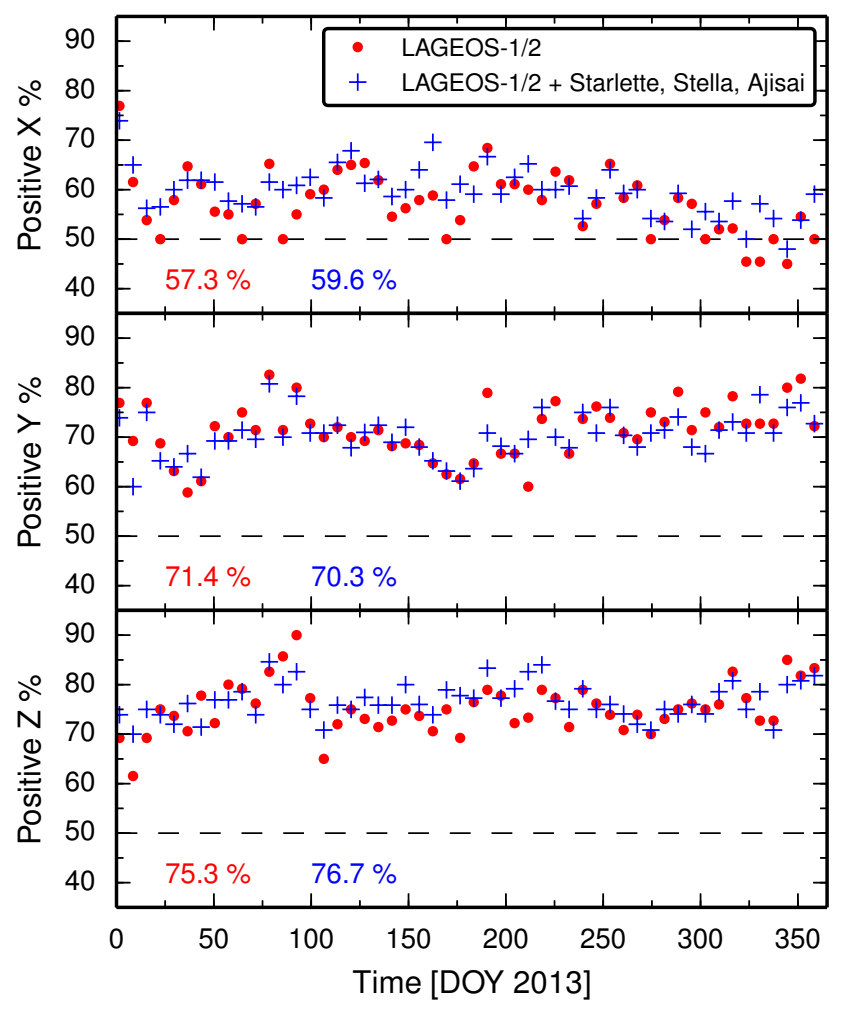

Fig. 2 Percentages of stations located in the hemispheres defined by the positive $X, Y$ and $Z$ Cartesian axes in LAGEOS-1 and 2 (type A) and LAGEOS-Starlette-Stella-Ajisai (type C) weekly solutions. Printed numerical values represent average percentages over the entire time span

spheres as a function of time for solutions of types A and C. In LAGEOS-only solutions, the network is biased towards the positive hemispheres and the incorporation of Starlette, Stella and Ajisai data further degrades the distribution along the $X$ and $Z$ axes. Figure 3 illustrates the variations over time in the number of stations and the number of NPs in solutions of types A, C and D. The contribution of the Etalon satellites is limited and therefore omitted from Fig. 3. Conversely, the addition of LEO data to LAGEOS data inflates the network size, but more significantly the number of measurements.

The geographical coverage of LAGEOS-1 and 2 observations is restricted mainly to continental regions and displays polar gaps (Fig. 4). On the other hand, Starlette, Stella, Ajisai and LARES NPs have a much more confined spatial extent owing to the lower altitudes of the satellites. Overall, the distribution of NPs is biased towards the northern hemisphere, despite the fact that the two most prolific stations in terms of data yield for the eight spherical geodetic satellites considered in this study are situated in the southern hemisphere (i.e. 7090 Yarragadee and 7825 Mount Stromlo). Together, 7090 and 7825 provide nearly one quarter of the data set. The third most prolific station is Zimmerwald (7810).

The main characteristics of the selected satellites are summarised in Table 1. All satellites, with the exception of Ajisai, are characterised by small area-to-mass ratios which reduce non-gravitational perturbations such as SRP and atmospheric drag. In particular, the area-to-mass ratio of LARES is approximately 2.6 times lower than that of each LAGEOS satellite, making LARES the densest known object in the Solar System (Paolozzi and Ciufolini 2013). Apart from atmospheric drag, the non-gravitational perturbations experienced by the orbit of LARES are typically the smallest in magnitude among all satellites. In contrast to LAGEOS-1 and 2, LARES is constructed of a solid single-piece sphere of smaller diameter and higher thermal conductivity. These particular physical characteristics minimise the thermal effects on LARES.

Ajisai contributes approximately $29 \%$ to the data set used in this study, more than the combined contribution of both LAGEOS satellites $(\sim 27 \%)$. The 1436 large cube corner reflectors aboard Ajisai ensure a high return rate of photons which, in conjunction with the favourable inclination for low- and mid-latitude tracking and longer satellite passes compared to Starlette and Stella, explains the prevalence of Ajisai NPs. Despite being the second largest targets and having the highest number of retro-reflectors, the contribution of the Etalon satellites amounts to less than $3 \%$ of the data set. Owing to their nearly equal inclinations, Starlette and Ajisai have similar perturbation spectra (Cheng et al 1997). Ajisai and LARES exhibit less sensitivity than Starlette and Stella to variations in the Earth's gravity field due to their higher altitudes. The quasi-polar orbit of Stella 

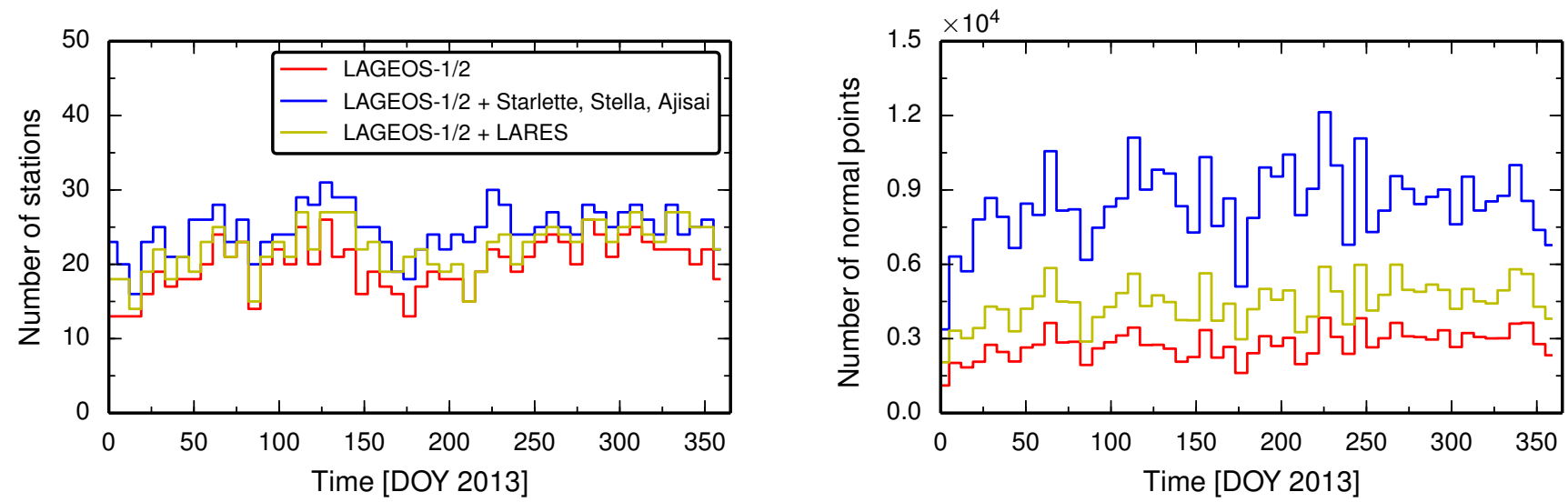

Fig. 3 Number of stations with more than 20 NPs (left $)$ and number of NPs (right) in LAGEOS-1 and 2 (type A), LAGEOS-Starlette-Stella-Ajisai (type C) and LAGEOS-LARES (type D) weekly solutions
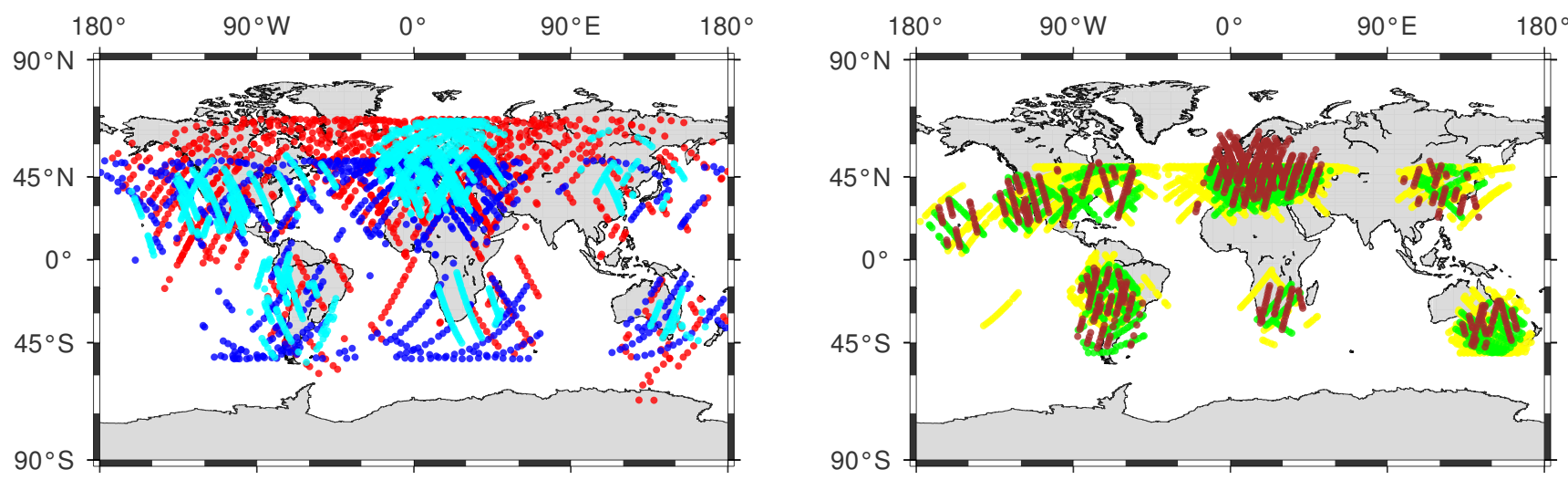

Fig. 4 Observed ground tracks of LAGEOS-1 (red circles), LAGEOS-2 (blue circles) and LARES (cyan circles) during the week 28 July - 3 August, 2013 (left). Observed ground tracks of Starlette (green circles), Stella (brown circles) and Ajisai (yellow circles) over the same time period (right)

was thought to positively influence the determination of the $Z$ geocentre coordinate (Sośnica et al 2014), but Kuang et al (2015) showed that the orbital inclination of $90^{\circ}$ is the least favourable for the observability of the $Z$ geocentre coordinate due to the poor information content of the tracking data in the axial direction.

Starlette, Stella, Ajisai and LARES are placed in low Earth orbits, which allow most SLR stations to track them on a regular basis. Despite their long tracking histories, Starlette, Stella and Ajisai are absent from the contributions submitted by the ILRS analysis centres (ACs) for the computation of the ITRF. The prime justifications for their omission are the large orbital perturbations related to temporal changes in the Earth's gravity field and the necessity to model atmospheric drag acting as a deceleration force on LEO orbits, mainly in the along-track direction. The drag force is a function of the atmospheric density at the satellite's position. Even though various thermospheric density models of different complexity are available (see, e.g., Montenbruck and Gill 2000), atmospheric density modelling remains a challenge in orbit determination since lim- ited progress has been achieved over the past few decades. NRLMSISE-00 (Picone et al 2002) is the model of choice in the current study.

Table 2 describes the models and conventions adopted for orbit determination and parameter estimation. These are in general agreement with the IERS Conventions (2010) (Petit and Luzum 2010) and the current processing standards of the ILRS ACs. System-dependent centre of mass (CoM) corrections (Otsubo and Appleby 2003) were applied for the LAGEOS, Etalon and Ajisai satellites, whereas for Starlette and Stella we adopted the value of $78 \mathrm{~mm}$ recommended by Ries (2008) rather than the standard value of $75 \mathrm{~mm}$. A recent independent study (Otsubo et al 2015) also reported an average Starlette and Stella CoM correction of 78-79 mm for the current SLR network. The standard CoM correction of $133 \mathrm{~mm}$ was applied to LARES range observations.

The chosen satellite-dependent scale factors (see Table 2) are equivalent to assigning a priori standard deviations of $1 \mathrm{~cm}$ to LAGEOS data, $1.5 \mathrm{~cm}$ to Etalon, $2.5 \mathrm{~cm}$ to Starlette and Stella, $3 \mathrm{~cm}$ to Ajisai and $2 \mathrm{~cm}$ to LARES. This weighting strategy was selected based on the typical post-fit 
Table 2 Data processing standards, models and conventions

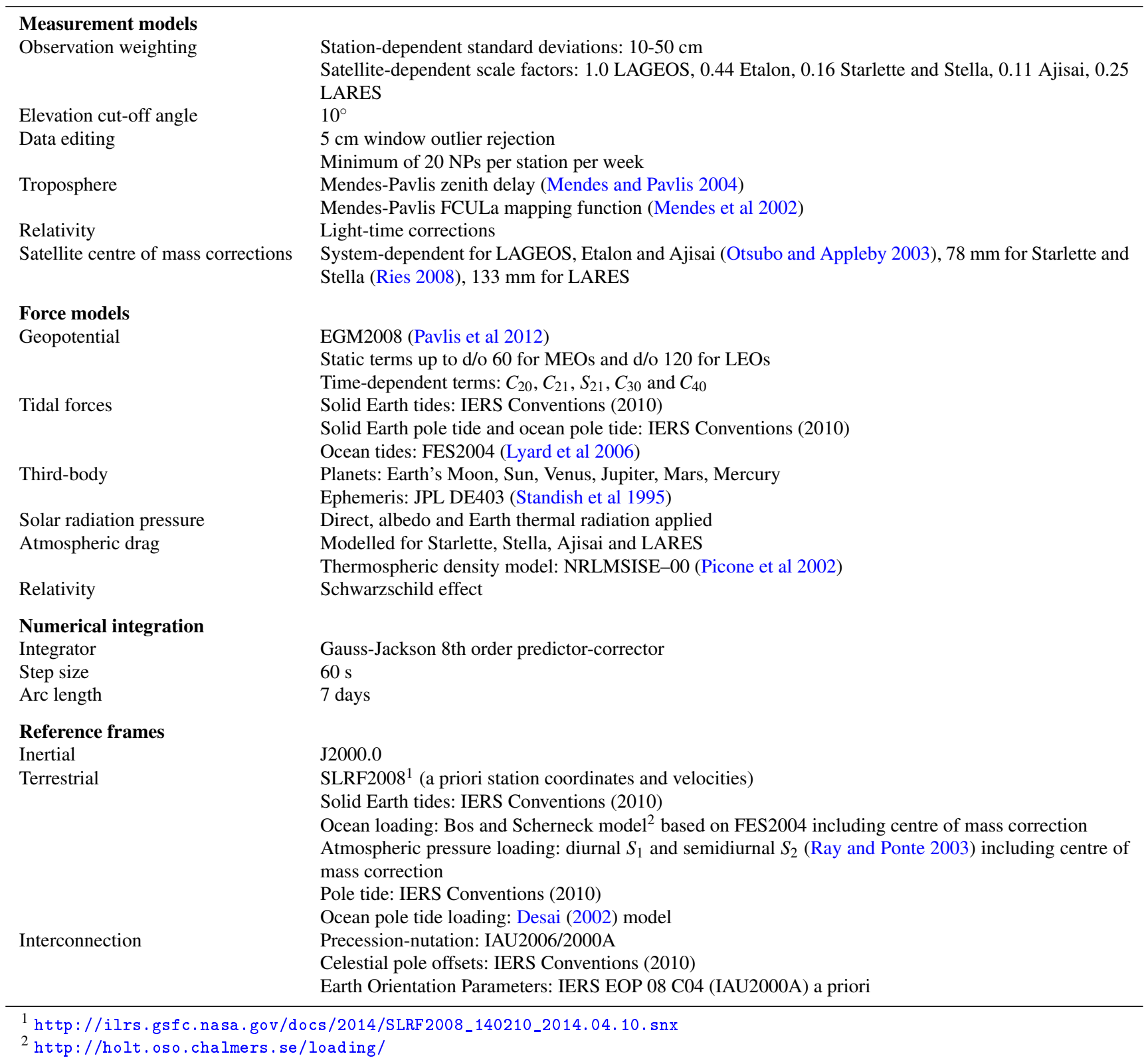

root mean square (RMS) of the observation residuals in an unweighted case. Downweighting LEO data is also desirable to counterbalance their dominance over MEO observations.

\subsection{Standard parameterisation}

The orbital parameterisations for MEOs and LEOs are described in Table 3, along with the estimated global parameters. The minimal set of parameters was sequentially supplemented by additional parameters to study the effects of various parameterisations on the observability of the geocentre coordinates, as described in Sect. 4 . The only difference between LEO and MEO orbits consists in the estima- tion of drag coefficients for LEO orbits in place of empirical along-track acceleration parameters. These two types of parameters are modelled using piecewise linear functions in Faust.

Unmodelled thermal drag forces acting on LAGEOS (Rubincam 1988, 1990) and the other satellites, as well as deficiencies in the modelling of direct and indirect SRP are accounted for by empirical along-track accelerations. The mismodelling of other non-conservative forces is compensated by OPR acceleration parameters in the along-track $\left(S_{s}\right.$, $\left.S_{c}\right)$ and cross-track $\left(W_{s}, W_{c}\right)$ directions. The cross-track OPR parameters also compensate for unmodelled variations in the Earth's gravity field and the mismodelled part of ocean tide 
constituents. There is a lack of consensus in the SLR community on the spacing of empirical parameters over time. ILRS ACs routinely parameterise LAGEOS solutions with either one or two sets of along-track and OPR along-track and cross-track accelerations per week.

Drag coefficients account mainly for the lack of precise knowledge of the atmospheric density in the upper atmosphere and are usually solved for either as piecewise constant or piecewise linear parameters. In a piecewise linear model, as employed in Faust, the first and last parameters are less well constrained by data than the middle parameters. An overparameterisation of an arc with unconstrained drag coefficients may thus lead to unrealistic negative values of the coefficients at the two ends. Parameterising a standard seven-day arc with only a pair of drag coefficients is equivalent to modelling drag as a linear function of time (i.e. drag and drag rate). Hereinafter, this practice will be referred to as weekly estimation of drag coefficients.

It is customary to set up daily (Cheng and Tapley 1999; Moore et al 2005; Maier et al 2012; Sośnica et al 2014) or even 12-hourly (Lejba and Schillak 2011; Cheng et al 2013) drag coefficients for LEO orbits to reduce the RMS of the observation residuals and hence improve the orbital fit. Solving for a high number of drag coefficients does, however, lead to increased correlations between parameters and potential collinearity issues. Despite lowering the residuals' RMS, such a routine casts doubt on the quality of the determined orbit and even the fidelity of the model with respect to physical reality. Using the RMS as a measure of the absolute quality of a solution is problematic, although it does offer insight into the relative difference in quality between two solutions.

In the current practice of the ILRS ACs, SRP coefficients are fixed to best-fit satellite-dependent values. Most ACs apply a value of 1.13 for both LAGEOS-1 and 2, but recent studies (Sośnica 2014; Zelensky et al 2014) indicated that a lower value of 1.10-1.11 better characterises the optical properties of LAGEOS-2, which may have changed since the launch of the satellite (Lucchesi et al 2004). We conformed to the practice of the ACs by fixing the SRP coefficients to the values listed in Table 1 in the standard parameterisation.

Pole coordinates and excess length of day (LOD) were estimated at noon of each day, whereas the UT1-UTC differences were fixed at noon to values obtained by linear interpolation of midnight offsets published by the International Earth Rotation and Reference Systems Service (IERS). Range biases were only estimated for certain stations suggested by the ILRS. ${ }^{4}$ The orientations of the estimated CM-centred frames were aligned with respect to the Satellite Laser Ranging Frame 2008 (SLRF2008) through

\footnotetext{
4 http://ilrs.dgfi.badw.de/fileadmin/data_handling/ ILRS_Data_Handling_File.snx
}

Table 3 Estimated orbital and global parameters in standard solutions

\begin{tabular}{lcc}
\hline Parameter & \multicolumn{2}{c}{ Spacing } \\
\hline Orbital parameters & MEOs & LEOs \\
Initial position and velocity & Weekly & Weekly \\
Drag coefficients & - & Weekly \\
Empirical along-track accelerations & Weekly & - \\
Empirical OPR along-track accelerations & Weekly & Weekly \\
Empirical OPR cross-track accelerations & Weekly & Weekly \\
Global parameters & & \\
Station coordinates & Weekly \\
Pole coordinates & Daily \\
Excess length of day & Daily \\
Range biases for selected stations & Weekly \\
\hline
\end{tabular}

no-net-rotation conditions applied over the core network in each weekly solution. Both orbital and global parameters were freely estimated.

\section{Results}

The simulations carried out by Rebischung et al (2014) using a synthetic SLR network yielded VIF values of 1.04 (20.4\%), $1.04(19.1 \%)$ and $8.6(94.0 \%)$ for the $X, Y$ and $Z$ geocentre coordinates, respectively. A 100-station uniformly distributed network with simulated observations every 5 minutes over 7 days to two LAGEOS-like satellites was used. However, this idealised network geometry and observation schedule are unrealistic for SLR. In this section, we extend the analysis to the actual SLR network and real data by introducing a $1 \mathrm{~cm}$ geocentre offset along each axis and applying the collinearity diagnostic procedure described in Sect. 2. To facilitate a direct comparison of the observability of the geocentre coordinates derived from different solution types, formal error values are provided in the following sections instead of VIFs. The formal error $1 /\left\|\mathbf{s}_{y}\right\|$ represents the theoretical uncertainty of each geocentre coordinate estimated in isolation of other parameters, whereas $1 /\left\|s_{y, c}^{p}\right\|$ is the actual uncertainty of each geocentre coordinate under independent parameter variations. The squared ratio of $1 /\left\|s_{y, c}^{p}\right\|$ and $1 /\left\|\mathbf{s}_{y}\right\|$ gives the VIF value.

\subsection{LAGEOS-1 and 2 solutions}

Seven solutions labelled A1 to A7 were produced for the LAGEOS- 1 and 2 combination. The differences in parameterisation with respect to the standard solution described in Table 3 are as follows:

- A1: no differences,

- A2: two sets of empirical along-track and OPR alongtrack and cross-track accelerations,

- A3: geopotential coefficients up to d/o 2, 
Table 4 Median formal errors of the geocentre coordinates derived from LAGEOS-1 and 2 (type A), LAGEOS-Etalon (type B), LAGEOSStarlette-Stella-Ajisai (type C) and LAGEOS-LARES (type D) solutions

\begin{tabular}{|c|c|c|c|c|c|c|}
\hline \multirow[t]{2}{*}{ Solution } & \multicolumn{3}{|c|}{ Median formal error $1 /\left\|\mathbf{s}_{y}\right\|[\mathrm{mm}]$} & \multicolumn{3}{|c|}{ Median formal error $1 /\left\|\mathbf{s}_{y, c}^{p}\right\|[\mathrm{mm}]$} \\
\hline & $X$ & $Y$ & $Z$ & $X$ & $Y$ & $Z$ \\
\hline A1 & 0.357 & 0.352 & 0.300 & 1.407 & 1.195 & 3.541 \\
\hline A2 & 0.358 & 0.352 & 0.300 & 1.443 & 1.177 & 3.503 \\
\hline A3 & 0.357 & 0.352 & 0.300 & 1.482 & 1.172 & 3.368 \\
\hline A4 & 0.357 & 0.352 & 0.300 & 1.529 & 1.216 & 3.493 \\
\hline A5 & 0.372 & 0.365 & 0.312 & 1.400 & 1.247 & 3.355 \\
\hline A6 & 0.357 & 0.352 & 0.300 & 1.528 & 1.238 & 3.722 \\
\hline A7 & 0.357 & 0.352 & 0.300 & 1.794 & 1.495 & 3.696 \\
\hline B1 & 0.342 & 0.335 & 0.284 & 1.354 & 1.161 & 3.442 \\
\hline B2 & 0.342 & 0.335 & 0.285 & 1.434 & 1.188 & 3.600 \\
\hline $\mathrm{C} 1$ & 0.224 & 0.225 & 0.196 & 0.821 & 0.724 & 1.932 \\
\hline $\mathrm{C} 2$ & 0.220 & 0.223 & 0.194 & 0.807 & 0.689 & 1.887 \\
\hline C3 & 0.221 & 0.223 & 0.195 & 0.811 & 0.715 & 1.874 \\
\hline $\mathrm{C} 4$ & 0.256 & 0.261 & 0.220 & 0.832 & 0.832 & 2.066 \\
\hline C5 & 0.222 & 0.222 & 0.194 & 1.629 & 1.010 & 3.547 \\
\hline C6 & 0.244 & 0.248 & 0.214 & 0.806 & 0.767 & 1.934 \\
\hline C7 & 0.243 & 0.245 & 0.214 & 0.871 & 0.817 & 2.069 \\
\hline $\mathrm{C} 8$ & 0.243 & 0.245 & 0.213 & 0.848 & 0.771 & 2.098 \\
\hline D1 & 0.282 & 0.280 & 0.238 & 6.594 & 5.148 & 15.902 \\
\hline D2 & 0.282 & 0.280 & 0.238 & 0.887 & 0.841 & 2.580 \\
\hline D3 & 0.282 & 0.280 & 0.238 & 0.910 & 0.892 & 2.582 \\
\hline D4 & 0.280 & 0.280 & 0.236 & 0.978 & 0.912 & 2.737 \\
\hline D5 & 0.280 & 0.279 & 0.236 & 1.037 & 0.888 & 2.801 \\
\hline D6 & 0.280 & 0.279 & 0.236 & 1.027 & 0.911 & 2.633 \\
\hline D7 & 0.280 & 0.279 & 0.236 & 1.110 & 0.940 & 2.873 \\
\hline
\end{tabular}

- A4: geopotential coefficients up to d/o 3,

- A5: geopotential coefficients up to $d /$ o 3 and no OPR accelerations,

- A6: geopotential coefficients up to d/o 3 and two sets of empirical accelerations, and

- A7: geopotential coefficients up to d/o 4 .

Only geopotential coefficients up to a maximum of degree 4 (excluding degree 1) were recovered given the insensitivity of LAGEOS-1 and 2 to harmonics of degree 5 and above (Cheng et al 1997; Moore et al 2005).

Weekly values of the formal errors were computed for each geocentre coordinate. Fluctuations in the values of the formal errors occur due to changes in network configuration, as well as variations in data coverage, quantity and quality. Table 4 contains the median formal errors over the considered 52-week time frame for each of the seven LAGEOS-1 and 2 solutions. The median values offer insight into the actual capability of the SLR technique to observe geocentre motion under different parameterisations. We will show that the parameterisation can indeed affect the observability of the geocentre coordinates in SLR solutions, just as in the case of the GNSS geocentre determination.

Solutions A1 and A2 closely follow the processing standards of the ILRS ACs. The mean RMS of observation residuals is $9.1 \mathrm{~mm}$ for LAGEOS-1 and $9.2 \mathrm{~mm}$ for LAGEOS-2 in solution A1, whereas in solution A2 the mean RMS is 8.5 $\mathrm{mm}$ for each of the two LAGEOS satellites. In addition to the improved orbital fit, the estimation of two sets of empirical accelerations per week instead of one set results in a reduced scatter of formal errors at the expense of only a minor increase in median formal error $1 /\left\|s_{y, c}^{p}\right\|$ of the $X$ geocentre coordinate. If geopotential coefficients are additionally recovered, the formal errors $1 /\left\|s_{y, c}^{p}\right\|$ of the equatorial geocentre coordinates are slightly amplified, particularly when OPR accelerations are also estimated (cf. solutions A4, A5 and A6). When geopotential coefficients beyond degree 3 are determined, the formal errors $1 /\left\|\mathbf{s}_{y, c}^{p}\right\|$ increase progressively, most likely due to the reduced sensitivity of the LAGEOS satellites to higher degree harmonics and the increased correlations between geopotential coefficients and OPR accelerations.

In solution $\mathrm{A} 1$, the parameters that experience the largest variations $-\boldsymbol{\gamma}_{y, c}$ in response to imposing a geocentre offset of $1 \mathrm{~cm}$ along any of the axis are, in order of magnitude, the following:

- the satellite initial state vectors, particularly the velocity components,

- OPR accelerations, mainly in the cross-track direction $\left(W_{s}, W_{c}\right)$, and

- station coordinates and range biases to similar extents. 
When determining geopotential coefficients, however, the OPR accelerations are the most affected parameters $\left(W_{s}\right.$ and $W_{c}$ in solution A3; $S_{s}$ and $W_{c}$ in solutions A4 and A7). The ability of OPR accelerations to absorb the signature of the geocentre offset increases with increasing degree of estimated harmonics. Nevertheless, OPR accelerations appear to play a minor role in LAGEOS-only solutions since neither their removal (solution A5) nor their increase in number (solution A6) greatly alter the formal error values.

Compared to the simulations conducted by Rebischung et al (2014), range biases were additionally estimated for selected stations in this study. We also processed the LAGEOS-1 and 2 data using the standard parameterisation but without solving for range biases. The median formal errors $1 /\left\|\mathbf{s}_{y, c}^{p}\right\|$ decreased by 5,3 and $10 \%$ for the $X, Y$ and $Z$ geocentre coordinates, respectively, with respect to solution A1. This result highlights the importance of both rigorous calibrations of tracking systems and system-dependent CoM corrections. The application of well-established range biases at the pre-processing level and of system-dependent CoM corrections for all spherical geodetic satellites may allow the removal of range biases from the estimated parameters and, thus, a more accurate determination of geocentre motion and potentially other parameters.

\subsection{LAGEOS-Etalon combined solutions}

The Etalon satellites are inserted in GNSS-like orbits which complicate their tracking and limit their sensitivity to temporal variations in the Earth's gravity field. In the current study, only 27 of the 34 contributing stations provided Etalon data. Despite being sparsely tracked, the Etalon satellites contribute to the definition of the ITRF origin. For completeness, they are thus included here to appraise their effect on the observability of the geocentre coordinates.

We generated two LAGEOS-Etalon combined solutions with the following differences with respect to the standard parameterisation:

- B1: no differences, and

- B2: geopotential coefficients up to d/o 3 .

In solution B1, the mean RMS of observation residuals is $12.2 \mathrm{~mm}$ for Etalon-1 and $11.8 \mathrm{~mm}$ for Etalon-2, whereas in solution B2 the orbital fits are $12 \mathrm{~mm}$ for Etalon-1 and $11.7 \mathrm{~mm}$ for Etalon-2. Table 4 lists the obtained median formal errors of the geocentre coordinates. Comparing solutions $\mathrm{B} 1$ to $\mathrm{A} 1$ and $\mathrm{B} 2$ to $\mathrm{A} 4$ reveals that the addition of Etalon observations to LAGEOS data has a limited effect on the observability of the geocentre coordinates. Etalon1 and 2 provide beneficial information for the determination of the geocentre location only when geopotential coefficients are omitted. The limited sensitivity of the Etalon satellites to time-varying gravity signals is reflected by the typically higher spreads of formal errors $1 /\left\|s_{y, c}^{p}\right\|$ in solution B2 relative to solutions A3-A7 and a degraded observability of the $Z$ geocentre coordinate. We consequently decided against including Etalon data in the MEO-LEO combined solutions described in Sections 4.3 and 4.4.

\subsection{LAGEOS-Starlette-Stella-Ajisai combined solutions}

The orbits of low Earth satellites Starlette, Stella and Ajisai are subject to large non-gravitational perturbations due to atmospheric drag, which pose difficulties for orbit modelling and restrict its accuracy. With the exception of geopotential coefficients, the geodetic products derived from Starlette, Stella and Ajisai data are typically of lower quality than their LAGEOS-derived equivalents (e.g. Lejba and Schillak 2011), but recent LAGEOS-Starlette-StellaAjisai data combinations involving complex modelling or LEO orbits yielded promising results (Cheng et al 2013; Sośnica et al 2014). However, to compensate for the effects of non-conservative forces and to improve the orbital fit to LAGEOS-like values, a large number of empirical parameters need to be solved for. Such parameters may also absorb geophysical signals in addition to modelling errors.

The orbits of Starlette, Stella and Ajisai are routinely parameterised with daily drag coefficients. To assess the consequences of such a practice on the observability of the geocentre coordinates in LAGEOS-Starlette-Stella-Ajisai combinations, we derived solutions with different temporal spacing of drag coefficients, ranging from one day to one week. As Fig. 5 (left) shows, median formal errors $1 /\left\|s_{y, c}^{p}\right\|$ increase exponentially as the number of drag coefficients increases. For each geocentre coordinate, over $98.8 \%$ of the variability in median formal errors $1 /\left\|\mathbf{s}_{y, c}^{p}\right\|$ can be explained by an exponential function of the type $a \exp (-b x)+c$ with coefficients $a, b$ and $c$. On the other hand, the orbital fit for Starlette, Stella and Ajisai is characterised by a gradual but limited improvement with increasing number of drag coefficients, as illustrated in Fig. 5 (right). The average differences in the RMS of the observation residuals between the solution with weekly drag coefficients and the solution with daily drag coefficients are 1.9, 0.5 and $1.1 \mathrm{~mm}$ for Starlette, Stella and Ajisai, respectively. Weekly differences are generally within $5 \mathrm{~mm}$ and sensibly lower for Ajisai (Fig. 6). The mean RMS of LAGEOS-1 and 2 observation residuals is at the level of $1 \mathrm{~cm}$, irrespective of the drag coefficient spacing.

In LAGEOS-Starlette-Stella-Ajisai combined solutions, the independent parameters most affected by the artificial geocentre offset are drag coefficients. The more estimated drag coefficients, the larger the variations and the better the drag coefficients are able to absorb the signatures the geocentre offsets on the observed ranges. Additionally, the first or first few drag coefficients are moderately to strongly correlated with the elements of the initial state vectors, which 

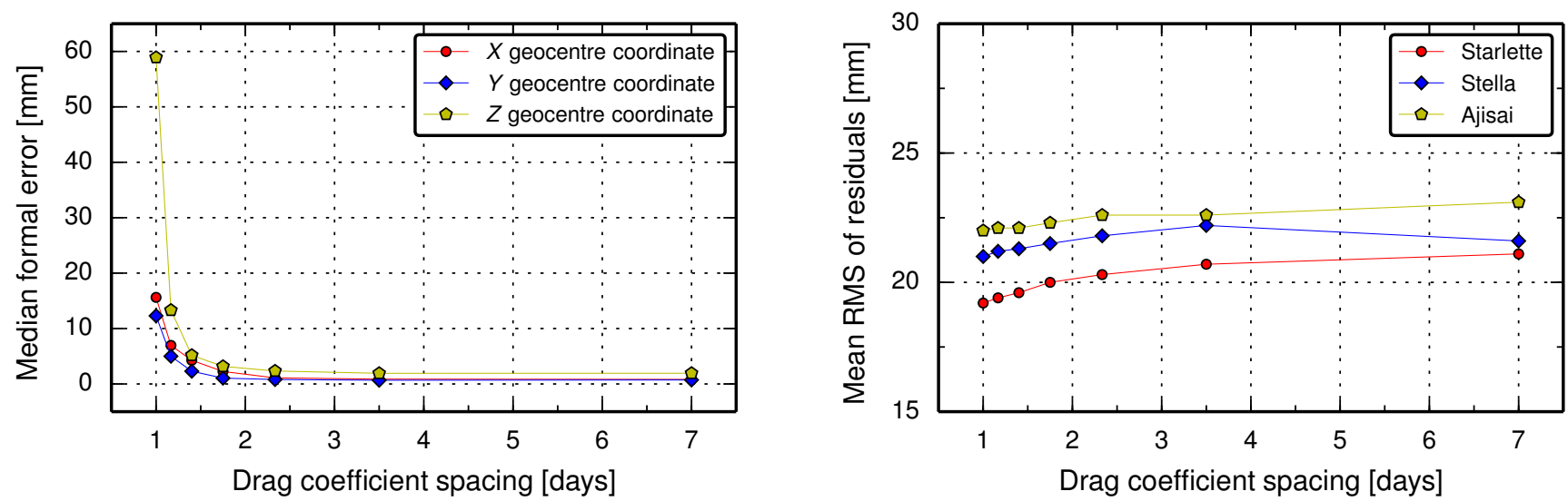

Fig. 5 Median formal errors $1 /\left\|s_{y, c}^{p}\right\|$ of the three geocentre coordinates as a function of drag coefficient spacing (left). Mean RMS of Starlette, Stella and Ajisai observation residuals as a function of drag coefficient spacing (right)

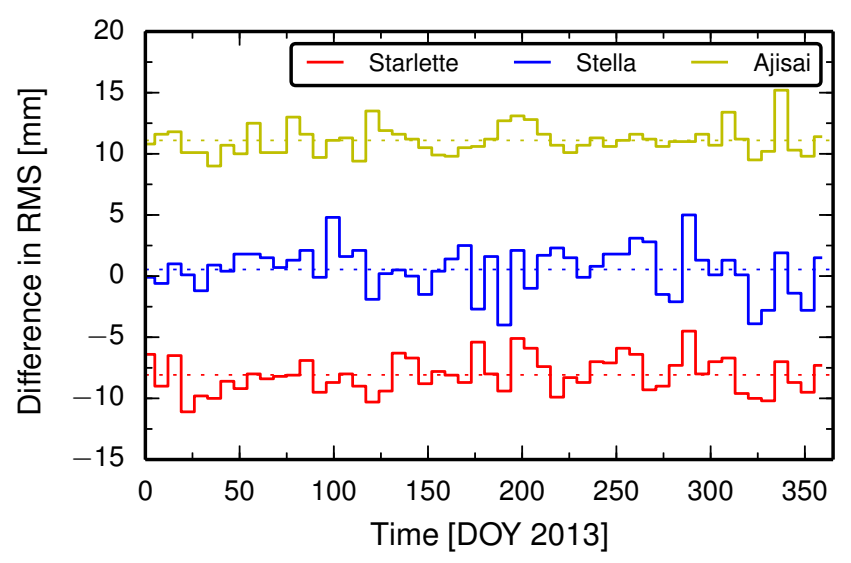

Fig. 6 Differences in the RMS of observation residuals between the solution with weekly drag coefficients and the solution with daily drag coefficients (weekly minus daily) for Starlette, Stella and Ajisai. Starlette and Ajisai values are shifted by $-10 \mathrm{~mm}$ and $+10 \mathrm{~mm}$, respectively. Dotted lines represent average differences

are the second most affected parameters by the introduced geocentre offset if geopotential coefficients are not estimated. These correlations also increase as the number of drag coefficients increases and play an important but secondary role in the collinearity issues of the geocentre coordinates with the other parameters in LAGEOS-StarletteStella-Ajisai solutions.

These findings led to the decision to parameterise Starlette, Stella and Ajisai orbits with weekly drag coefficients in the remainder of the analysis to isolate the collinearity problems induced by drag coefficients. To investigate the influence of other parameters on the observability of the geocentre coordinates, eight solutions labelled $\mathrm{C} 1$ to C8 were produced. They display the following differences with respect to the standard parameterisation:

- C1: no differences,

- C2: two sets of empirical along-track and OPR alongtrack and cross-track accelerations for LAGEOS orbits and two sets of OPR along-track and cross-track accelerations for LEO orbits,

- C3: geopotential coefficients up to $\mathrm{d} / \mathrm{o} 2$,

- C4: geopotential coefficients up to $d / o$ and no OPR accelerations for LEO orbits only,

- C5: geopotential coefficients up to d/o 3,

- C6: geopotential coefficients up to d/o 3 and no OPR accelerations for LEO orbits only,

- C7: geopotential coefficients up to d/o 4 and no OPR accelerations for LEO orbits only, and

- C8: geopotential coefficients up to d/o 5 and no OPR accelerations for LEO orbits only.

Starlette, Stella and Ajisai are sensitive to harmonic coefficients beyond degree 20 (Cheng et al 1997; Moore et al 2005). Our analysis was, however, restricted to degree 5 following the conclusion of Maier et al (2012) that the estimation of geopotential coefficients above degree 5 leads to ill-conditioned systems of normal equations.

The computed median formal errors of the three geocentre coordinates are given in Table 4 . In addition to the lower spread of values, the median formal error $1 /\left\|\mathbf{s}_{y, c}^{p}\right\|$ of any geocentre coordinate is typically smaller by 40 to $45 \%$ in LAGEOS-Starlette-Stella-Ajisai solutions compared to LAGEOS-only. Since the source of the improvement is not an ameliorated station distribution (see Fig. 2) and the formal errors $1 /\left\|s_{y, c}^{p}\right\|$ are only weakly correlated with the network size and moderately correlated with the number of observations, the intrinsic sensitivity of Starlette, Stella and Ajisai to geocentre motion is partly responsible for the enhanced observability of the geocentre coordinates. Unlike the formal errors $1 /\left\|s_{y, c}^{p}\right\|$, the values of $1 /\left\|s_{y}\right\|$ are very strongly correlated with the size of the data set. The direction of the correlation is negative in both cases.

As previously mentioned, drag coefficients exhibit the largest variations $-\boldsymbol{\gamma}_{y, c}$ following the introduction of an artificial geocentre offset of $1 \mathrm{~cm}$ along any Cartesian axis. 

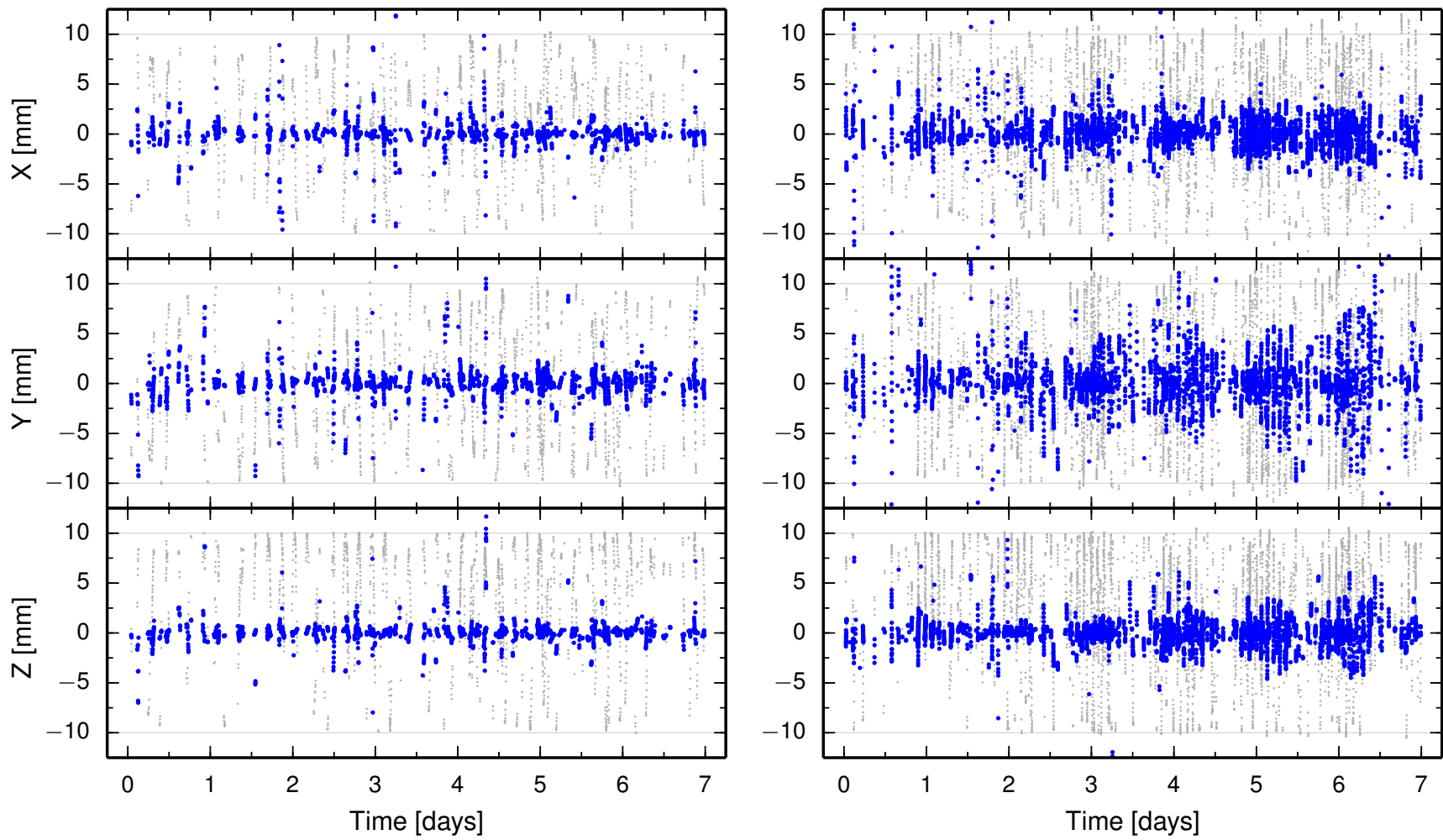

Fig. 7 Signature $\mathbf{s}_{y}$ (grey circles) and proper signature $\mathbf{s}_{y, c}^{p}$ (blue circles) of a $1 \mathrm{~cm}$ geocentre offset along each axis on ranges to LAGEOS-1 and 2 (left) and ranges to Starlette, Stella and Ajisai (right). Plotted data are for the arcs spanning the week 28 July - 3 August, 2013 and solution C1

In solution $\mathrm{C} 3$, the second most affected parameters are the OPR acceleration components $W_{c}$, whereas in solution C5 both $S_{s}$ and $W_{c}$ experience large variations. Increasing the degree of estimated geopotential coefficients inflates the variations of drag coefficients and OPR accelerations, thus enhancing their ability to jointly compensate the geocentre offsets (see, e.g., solution C5). Nevertheless, omitting the OPR accelerations for the Starlette, Stella and Ajisai when estimating geopotential coefficients greatly reduces the collinearity problems of the geocentre coordinates, as illustrated by solutions C6, C7 and C8. Hence, we conclude that the simultaneous determination of OPR accelerations for Starlette, Stella and Ajisai and geopotential coefficients above degree 2 reduces the observability of the geocentre coordinates in LAGEOS-Starlette-Stella-Ajisai solutions due to the correlations between the OPR terms, mainly in the cross-track direction, and the geopotential coefficients.

An alternative parameterisation to $\mathrm{C} 1$ with weekly drag coefficients fixed to the widely used value of 2.2 (see, e.g., Cook 1965) and weekly empirical along-track accelerations was performed, but resulted in only a small improvement $(\sim 5 \%)$ in the formal errors $1 /\left\|s_{y, c}^{p}\right\|$ over solution $\mathrm{C} 1$. Using such a parameterisation, however, the RMS of observation residuals can be reduced for Starlette, Stella and Ajisai by reasonably increasing the number of empirical accelerations without the risk of compromising the observability of the geocentre coordinates when geopotential coefficients are omitted.

Independent parameter variations are less able to absorb the signature of the geocentre offset on ranges to Starlette, Stella and Ajisai than on ranges to LAGEOS-1 and 2. Figure 7 presents the case of the weekly combined solution with the lowest formal errors $1 /\left\|s_{y, c}^{p}\right\|$ for all three geocentre coordinates. The spatial coverage of NPs during the same week is shown in Fig. 4. In all weekly solutions of type C, with the exception of $\mathrm{C} 5$, the proper signature is larger for Starlette, Stella and Ajisai ranges than for LAGEOS-1 and 2 ranges. This constitutes evidence of the higher sensitivity of LEOs to geocentre motion.

Observations to Starlette, Stella and Ajisai were also separately integrated with LAGEOS- 1 and 2 data to appraise the relative contribution of each LEO to combined solutions. The standard parameterisation was adopted for both LAGEOS and LEO orbits. The combination of Starlette with LAGEOS- 1 and 2 provides the most stable results (i.e. the lowest spread of formal errors $\left.1 /\left\|s_{y, c}^{p}\right\|\right)$. Ajisai appears to contribute the most to the observability of the $Z$ geocentre coordinate rather than Stella, as argued by Sośnica et al (2014). This result is partly due to the large number of Ajisai observations, but confirms through real data analysis the finding of Kuang et al (2015) that a polar orbit is the least favourable for the determination of the $Z$ geocentre coordi- 


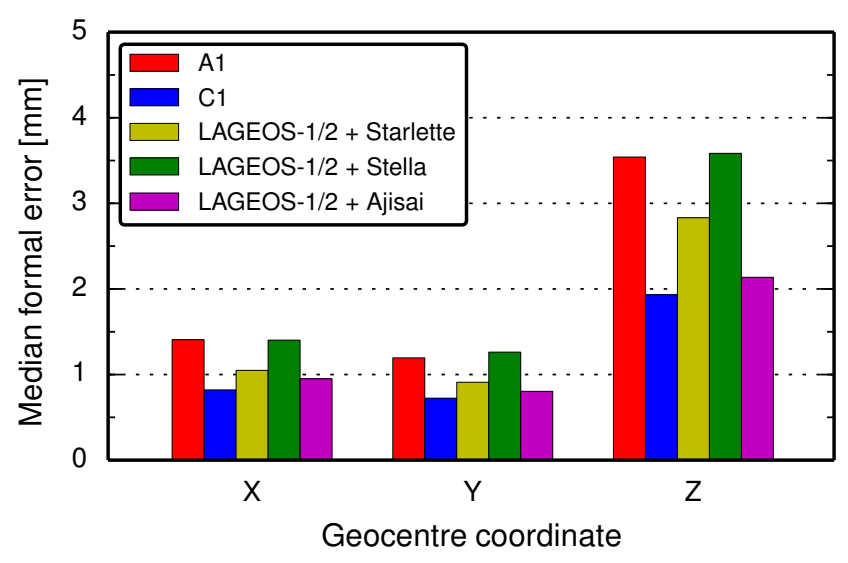

Fig. 8 Median formal errors $1 /\left\|s_{y, c}^{p}\right\|$ of the three geocentre coordinates in solutions A1, C1 and separate combinations of Starlette, Stella and Ajisai with LAGEOS-1 and 2

nate. Kuang et al (2015) obtained this result using synthetic SLR data from a network of 40 stations tracking a single box-wing LEO and estimating an SRP coefficient, which is strongly correlated with the $Z$ component of the geocentre vector (see, e.g., Meindl et al 2013). For polar orbits, the $Z$ geocentre vector component lies in the orbital plane and can be compensated by the in-plane (i.e. radial and alongtrack) periodic orbital perturbations induced by SRP. Our study shows that this statement holds even when the SRP coefficients are fixed, since Stella has an insignificant contribution to the observability of the geocentre coordinates compared to Starlette, despite the similar construction and altitudes of the two satellites. The quasi-polar orbit of Stella also hampers its tracking by the current SLR network that abounds in stations located at low and medium latitudes.

The observability of the geocentre coordinates is improved by combining LAGEOS-1 and 2 with Starlette or Ajisai, whereas Stella fails to contribute significantly. No single LEO can fully explain the reduction in formal errors $1 /\left\|s_{y, c}^{p}\right\|$ and their spread from solution A1 to C1 (Fig. 8). Important factors for the uncertainty reduction are the larger number of observations and the higher sensitivity of LEOs to geocentre motion.

\subsection{LAGEOS-LARES combined solutions}

Among the LEOs considered in this study, LARES is arguably the most suitable target for TRF determination given its favourable design features. As the inclusion of LARES in the ILRS operational products is imminent, we derived seven LAGEOS-LARES solutions to investigate the potential benefits of incorporating LARES data for the origin of the TRF. The seven solutions display the following differences with respect to the standard parameterisation:

- D1: no differences,

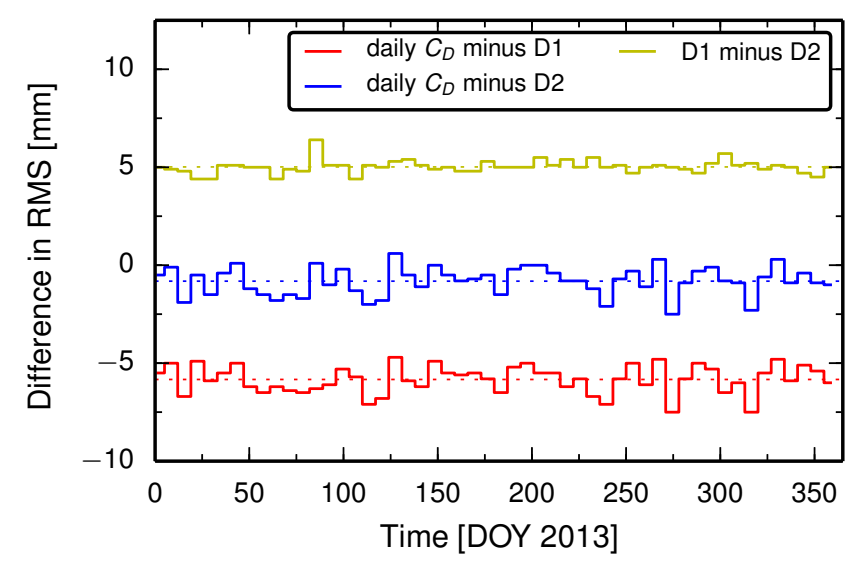

Fig. 9 Differences in the RMS of LARES observation residuals between the solution with daily drag coefficients (designated as daily $C_{D}$ ) and solutions D1 and D2. Daily $C_{D}$ minus D1 and D1 minus D2 values are shifted by $-5 \mathrm{~mm}$ and $+5 \mathrm{~mm}$, respectively. Dotted lines represent average differences

- D2: one set of empirical along-track accelerations for LARES orbits instead of drag coefficients,

- D3: two sets of empirical along-track and OPR alongtrack and cross-track accelerations for all orbits,

- D4: one set of empirical along-track accelerations for LARES orbits and geopotential coefficients up to d/o 2,

- D5: one set of empirical along-track accelerations for LARES orbits and geopotential coefficients up to d/o 3,

- D6: one set of empirical along-track accelerations for LARES orbits and geopotential coefficients up to d/o 4, and

- D7: one set of empirical along-track accelerations for LARES orbits and geopotential coefficients up to d/o 5 .

The mean RMS of LARES observation residuals improves from $18.8 \mathrm{~mm}$ in solutions D1 and D2 to $13.1 \mathrm{~mm}$ in solution D7, whereas the orbital fits of LAGEOS-1 and 2 observations lie in the confined interval $8.5-9.7 \mathrm{~mm}$, with the lowest value in solution D7. Table 4 contains the computed median formal errors of the geocentre coordinates in solutions of type D.

In solution D1, drag coefficients vary the most among all parameters after the introduction of a $1 \mathrm{~cm}$ geocentre offset along any axis. The variations are larger than in type $\mathrm{C}$ solution and can compensate the artificial geocentre offset to a great extent, even when drag coefficients are estimated weekly rather than daily. The orbital perturbations due to atmospheric drag are smaller in magnitude for LARES than for any other LEO included in this study. This allows the efficient modelling of atmospheric drag effects on LARES using a single set of empirical along-track acceleration parameters instead of drag coefficients, as illustrated in Fig. 9 by the small differences in the RMS of observations residuals between the alternative parameterisations D1 and D2 and a solution with daily drag coefficients for LARES or- 
bits. The next most affected parameters in solution D1 after drag coefficients are the velocity components of the initial state vectors, followed by station coordinates.

The parameters displaying the largest variations $-\boldsymbol{\gamma}_{y, c}$ in solution D2 are the same as in solution A1, namely the satellite initial state vectors, the OPR acceleration parameters $W_{s}$ and $W_{c}$, station coordinates and range biases. Similarly to LAGEOS-only solutions, when geopotential coefficients are also estimated, the OPR accelerations are the most affected parameters $\left(W_{c}\right.$ in solution D4; $S_{s}$ and $W_{c}$ in solutions D5, D6 and D7), followed by the satellite velocity components. Unlike in type $\mathrm{C}$ solutions, the observability of the geocentre coordinates is unaffected by the simultaneous determination of OPR accelerations for LARES and geopotential coefficients beyond degree 2 (cf. solutions C5 and D5). In an alternative solution to D1 and D2 with weekly drag coefficients fixed to the value of 2.2 for LARES and weekly empirical along-track accelerations, the orbital fits are identical to solutions D1 and D2 for all three satellites, whereas the observability of the geocentre vector is largely unaffected.

The median formal errors $1 /\left\|s_{y, c}^{p}\right\|$ of the geocentre coordinates are generally smaller by 20 to $35 \%$ in LAGEOSLARES solutions relative to LAGEOS-only and LAGEOSEtalon. Given the low altitude of LARES, the estimation of geopotential coefficients in LAGEOS-LARES solutions may be necessary to account for unmodelled and mismodelled long-wavelength variations in the Earth's gravity field. For the sake of an accurate determination of the geocentre location, a maximum truncation degree of 4 is preferable. The degraded observability of the geocentre coordinates in LAGEOS-LARES solutions with estimated geopotential coefficients beyond degree 4 (see solution D7) can be attributed to the insensitivity of LAGEOS-1 and 2 to these harmonics.

In combination with LAGEOS and other LEOs, LARES may also contribute to the separation of correlated geopotential coefficients. Preliminary analyses performed by Ciufolini et al (2012) demonstrated a significant reduction in the uncertainties of geopotential coefficients determined from LARES data compared to observations of other spherical geodetic satellites.

\subsection{Effects of solar radiation pressure modelling}

Direct SRP represents the dominant source of nongravitational perturbations for the orbits of Ajisai, LARES and satellites at higher altitudes. Atmospheric drag may exert larger perturbations than direct SRP on the orbits of Starlette and Stella. For spherical geodetic satellites, the acceleration due to direct SRP is more accurately modelled than the acceleration due to atmospheric drag owing to the lower uncertainty of the SRP force model components (see, e.g., Milani et al 1987). In addition, Gobinddass et al

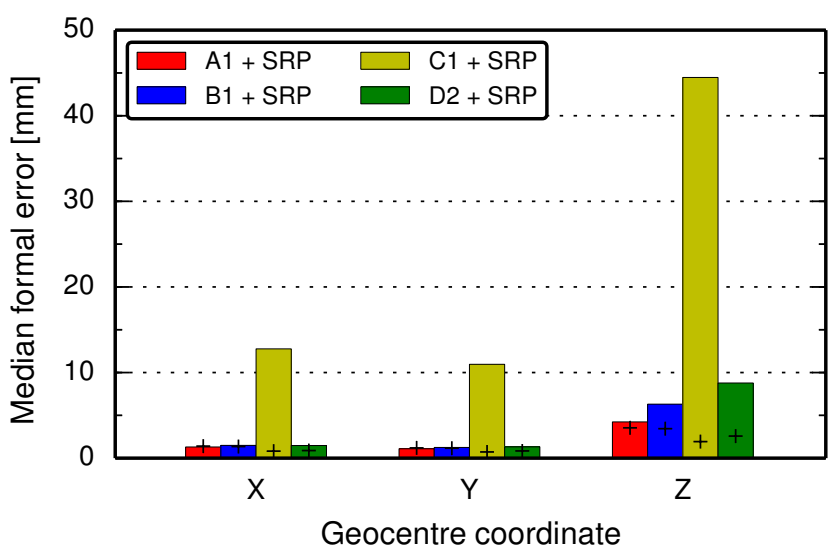

Fig. 10 Median formal errors $1 /\left\|\mathbf{s}_{y, c}^{p}\right\|$ of the three geocentre coordinates when the SRP coefficients of all satellites are estimated instead of being fixed in solutions A1, B1, C1 and D2. The median formal errors of the geocentre coordinates in solutions A1, B1, C1 and D2 are plotted as black pluses for comparison

(2009a) and Meindl et al (2013) showed that the effects of SRP mismodelling on the estimated geocentre coordinates are much less problematic for laser ranging to spherical geodetic satellites than for DORIS and GNSS. In SLR analyses, SRP coefficients are commonly held fixed to satellitespecific a priori values, as done throughout this study. SRP introduces periodic orbital perturbations only in the radial and along-track directions. The along-track OPR acceleration parameters $S_{S}$ and $S_{c}$ are correlated with the SRP coefficients and can compensate the mismodelled part of SRP to a large extent. SRP modelling deficiencies are typically reflected in the power spectra of the geocentre coordinates by anomalous harmonics occurring at periods corresponding to the draconitic years of the spherical geodetic satellites, as well as at multiples of the draconitic frequencies.

To investigate the effects of the correlation between SRP coefficients and along-track OPR acceleration parameters on the observability of the geocentre coordinates, we adopted the parameterisations from solutions A1, B1, C1 and D2, but freely estimated the SRP coefficient for each satellite. As illustrated in Fig. 10 via median formal errors $1 /\left\|s_{y, c}^{p}\right\|$, SRP coefficients and along-track OPR acceleration parameters have the potential to absorb the fictitious geocentre offset and thus reduce the observability of the geocentre vector, in particular of the $Z$ component. The reduction in observability is more prominent in multi-satellite combinations (especially LAGEOS-Starlette-Stella-Ajisai) due to the larger variations of the SRP coefficients of LEOs. Fixing the SRP coefficients to a priori values can alleviate this collinearity issue without significant compromise in orbital fit. Omitting the estimation of along-track OPR terms is another but less justifiable option. 


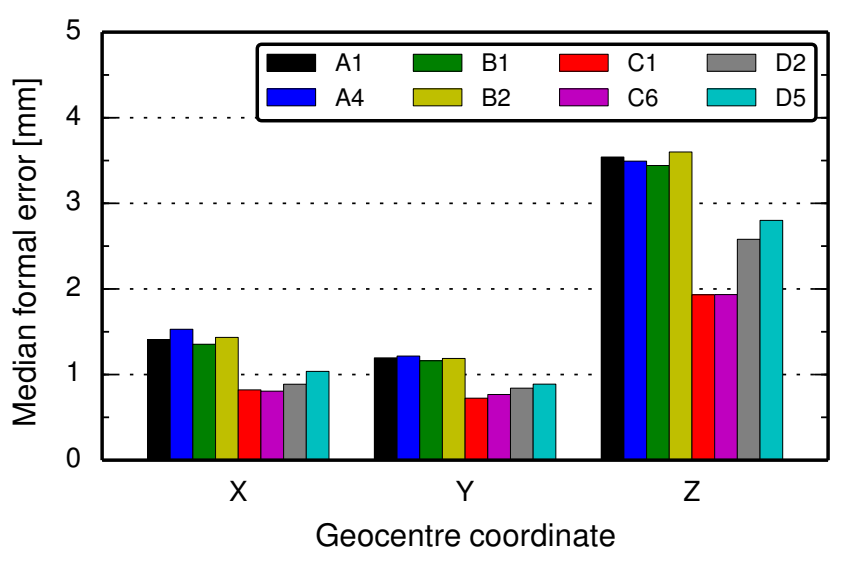

Fig. 11 Comparison of median formal errors $1 /\left\|s_{y, c}^{p}\right\|$ obtained for the three geocentre coordinates in eight different solutions of types A (LAGEOS-1 and 2), B (LAGEOS-Etalon), C (LAGEOS-StarletteStella-Ajisai) and D (LAGEOS-LARES)

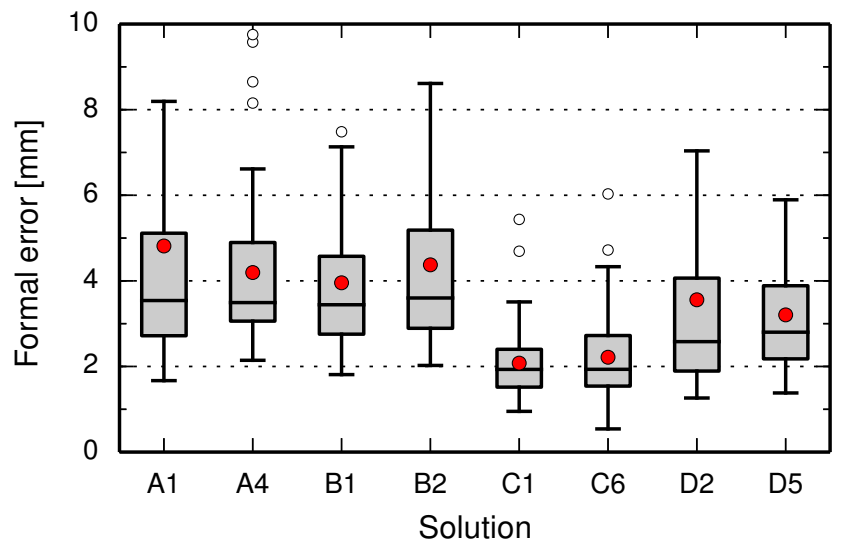

Fig. 12 Box plots displaying the 25th $\left(Q_{1}\right), 50$ th (median) and 75th $\left(Q_{3}\right)$ percentiles and the 1.5 interquartile ranges $\left(I Q R=Q_{3}-Q_{1}\right)$ of the formal errors $1 /\left\|s_{y, c}^{p}\right\|$ computed for the $Z$ geocentre coordinate in six different solutions. Mean formal errors are represented by red circles and outlying formal errors (i.e. larger than $Q_{1}+1.5 \cdot I Q R$ in this case) are plotted as hollow circles

\subsection{Comparison of solutions}

Although not theoretically expected, variations in the formal error $1 /\left\|\mathbf{s}_{y}\right\|$ among solutions of the same type (see Table 4) are due to the different number of accepted observations following the rejection of outliers. Low values of $1 /\left\|\mathbf{s}_{y}\right\|$ are indicative of a high acceptance rate and vice versa. Solving for a high number of empirical parameters typically increases the acceptance rate (cf. solutions C3 and C4, also C5 and C6). A similar effect of the number of accepted observations on the formal error $1 /\left\|s_{y, c}^{p}\right\|$ is expected, but the statistical relationships between parameters are the main driver of fluctuations in $1 /\left\|s_{y, c}^{p}\right\|$ numerical values.

The median formal errors $1 /\left\|s_{y, c}^{p}\right\|$ obtained for the geocentre coordinates in six of the most representative solutions of types A, B, C and D are compared in Fig. 11. In
LAGEOS-Etalon combinations, median formal errors are marginally lower than in LAGEOS-only solutions, except for the formal error of the $Z$ geocentre coordinates when geopotential coefficients are also recovered. Combining LAGEOS- 1 and 2 with Starlette, Stella and Ajisai or only with LARES significantly reduces the median formal errors, particularly for the $Z$ geocentre coordinate. By using only observations acquired by the core network shown in Fig. 1 and the standard parameterisation, the median formal errors $1 /\left\|s_{y, c}^{p}\right\|$ are largely unaltered for any data combination. This approach results in improved network distributions at the expense of greatly diminished network sizes and data coverage.

The box plots of the $Z$ coordinate formal errors $1 /\left\|s_{y, c}^{p}\right\|$ shown in Fig. 12 illustrate the differences in the observability of this component in the eight solutions compared in Fig. 11. Solutions A1 and D2 contain outliers which notably shift the arithmetic means away from the medians. Estimating geopotential coefficients in LAGEOS-only and LAGEOS-LARES solutions reduces the scatter of formal errors, but a similar effect can be achieved by doubling the number of empirical acceleration parameters. The relative improvement in observability from solutions of types A-B to solutions of types $\mathrm{C}-\mathrm{D}$ is indicated by the lower placement of the box plots for the latter. The distribution of formal errors is generally skewed towards large values, which explains the larger averages compared to the medians.

Among the two equatorial components of the geocentre vector, $X$ is less observable in SLR data analyses involving any combination of observations to spherical geodetic satellites, potentially due to its higher sensitivity to network effects (Collilieux et al 2009). The considerably stronger collinearity of the $Z$ coordinate with other parameters engenders an increase of its formal error by a factor of 2-3 compared to the formal errors of the $X$ and $Y$ geocentre coordinates. The geocentre coordinates are typically more observable in LAGEOS-LEO combinations than in LAGEOSonly solutions due to both the higher sensitivity of LEOs to geocentre motion and the larger number of observations.

To validate the inferences from the analysis of formal errors, we derived time series of geocentre coordinates from the eight solutions compared in Fig. 11 using the network shift approach with no scale factors estimated between weekly CM-centred frames and SLRF2008. Figure 13 depicts the unfiltered series from the LAGEOS-only, LAGEOS-Starlette-Stella-Ajisai and LAGEOS-LARES solutions. Due to their similarity with the LAGEOS-only series and to avoid clutter, the LAGEOS-Etalon series are omitted from Fig. 13. The RMS of the differences between the LAGEOS-only and LAGEOS-Etalon series derived from solutions with identical parameterisation varies between 0.9 $\mathrm{mm}$ and $1.6 \mathrm{~mm}$, depending on the geocentre coordinate. 

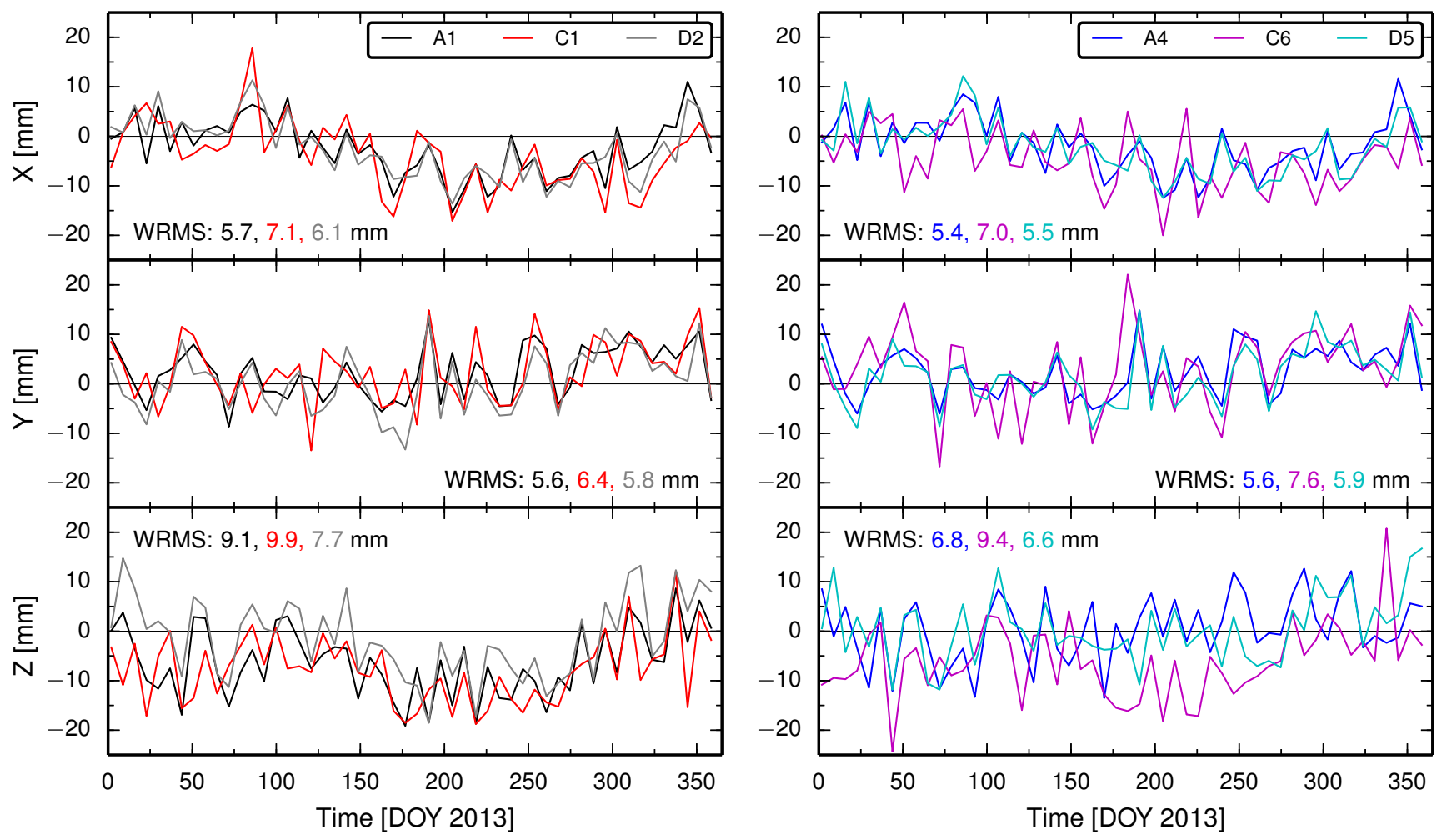

Fig. 13 Unfiltered geocentre coordinates obtained via the network shift approach from solutions A1, C1 and D2 (left) and solutions A4, C5 and D5 (right). Six parameters (i.e. three translations and three rotations) were estimated weekly with respect to SLRF2008

This casts further doubt on the value added by Etalon data in LAGEOS-Etalon combinations.

The scatter of the geocentre motion series, quantified through the weighted root mean square (WRMS) of the geocentre coordinates, is typically lower in solutions with estimated geopotential coefficients. This is particularly true for the $Z$ geocentre coordinate, which experiences a reduction in scatter of up to $26 \%$ in LAGEOS-only solutions. However, the annual variation of the axial geocentre motion component appears to be absorbed by the estimated geopotential coefficients, possibly due to $Z$ being exclusively determined by orbital perturbations due to the geopotential (Angermann and Müller 2008). Both the geopotential and Earth rotation affect the equatorial geocentre coordinates.

The LAGEOS-Starlette-Stella-Ajisai series exhibit the largest high-frequency variations and consequently have the largest scatter. Orbit modelling deficiencies may have contaminated the geocentre coordinates estimated from type $\mathrm{C}$ solutions given the simplistic force modelling applied for Starlette, Stella and Ajisai to circumvent collinearity issues. Despite the strong collinearity of the geocentre coordinates, the solution with daily drag coefficients estimated for Starlette, Stella and Ajisai (not shown in Fig. 13) is very similar to solution $\mathrm{C}$, but still noisier than other types of solutions. Without significant advances in surface force modelling, a beneficial contribution of Starlette, Stella and Ajisai to the definition of the ITRF origin appears unlikely, despite the improved observability of the geocentre coordinates.

The estimates of the equatorial geocentre coordinates from LAGEOS-only and LAGEOS-LARES solutions are in good agreement, despite the marginally larger scatter of the LAGEOS-LARES series. The WRMS of the $Z$ geocentre coordinates is, nevertheless, lower in LAGEOS-LARES solutions, suggesting a potential improvement relative to LAGEOS-1 and 2 solutions. More conclusive evidence should be provided by the analysis of longer times series, which may constitute the topic of a future study.

\section{Discussion and conclusions}

By means of collinearity diagnosis, this study set out to determine the actual current capability of SLR to sense geocentre motion and whether observations to the Etalon satellites and the LEOs Starlette, Stella, Ajisai and LARES can improve the observability of the geocentre coordinates with respect to standard LAGEOS-1 and 2 solutions. The processing of LAGEOS and Etalon NP data closely followed the standards of the ILRS ACs and the IERS Conventions (2010) (Petit and Luzum 2010). Numerous solutions involving various data combinations were generated to investigate the effect of different parameterisations on the collinearity 
of the geocentre coordinates with the other parameters in the analyses.

Under ideal conditions, the determination of the $X$ and $Y$ geocentre coordinates from simulated observations to LAGEOS-like satellites is free of collinearity issues, whereas the determination of the $Z$ coordinate suffers minor collinearity issues. We showed that under real world conditions, the determination of the $X$ and $Y$ coordinates also suffers minor collinearity problems, whereas the $Z$ coordinate is less observable by a factor of $2-3$. The collinearity problems of the geocentre coordinates obtained from real SLR data are largely attributable to network effects and the unevenly spaced and imperfect observations of heterogeneous quality. The estimation of geopotential coefficients beyond degree and order 3 further amplifies the collinearity issues in LAGEOS-only solutions.

The combination of LAGEOS observations with Etalon data has a marginally positive effect on the observability of the geocentre coordinates only when geopotential coefficients are omitted. One reason for this result is that, in LAGEOS-Etalon solutions, the doubling of the number of orbital parameters (leading to increased correlations between parameters) is only accompanied by an increase of a few percent $(\sim 10 \%)$ in the size of data set. We thus consider the incorporation of Etalon data in SLR analyses to be questionable for the purpose of geocentre motion determination using the network shift approach.

Observations to the low altitude satellites Starlette, Stella and Ajisai can potentially improve the ties of the SLR-derived weekly TRFs to the CM frame subject to two parameterisation constraints. First, the parameterisation of orbital arcs of Starlette, Stella and Ajisai with frequent (i.e. sub-weekly) drag coefficients has a detrimental effect on the observability of the geocentre coordinates in LAGEOS-Starlette-Stella-Ajisai combined solutions. Second, the simultaneous estimation of OPR acceleration parameters for Starlette, Stella and Ajisai and geopotential coefficients above degree 2 also negatively affects the determination of geocentre motion, but on a smaller scale than frequently spaced drag coefficients. A third parameterisation constraint applies to all satellite combinations, but especially to LAGEOS-Starlette-Stella-Ajisai. The concurrent determination of SRP coefficients and along-track OPR acceleration parameters increases the formal errors of the geocentre coordinates due to the well-known correlations between the two types of parameters. Despite these collinearity issues, the real geophysical signal is expected to be recoverable in multi-satellite solutions involving LEOs.

The larger proper signature on Starlette, Stella and Ajisai ranges compared to LAGEOS ranges constitutes evidence of the higher sensitivity of LEOs to geocentre motion. The $Z$ component of geocentre motion benefits most in an absolute sense from the combination of LAGEOS and LEO data. However, the geocentre coordinates estimated from LAGEOS-Starlette-Stella-Ajisai solutions are likely to be contaminated by force modelling errors that cannot be accommodated using a simplistic modelling strategy required to avoid collinearity issues. Until a satisfactory compromise between minimising orbital errors and circumventing collinearity issues is found, the exploitation of the high sensitivity of Starlette and Ajisai to geocentre motion appears unlikely. The sensitivity of Stella is lower due to its quasipolar orbit, which also negatively affects the tracking of the satellite by the current SLR network.

Drag coefficients are predominantly used to absorb errors in atmospheric density modelling. Thus, an obvious path to more accurate geocentre motion estimates from LAGEOS-Starlette-Stella-Ajisai combinations is significant improvements of atmospheric models. Such developments would, in turn, allow the routine estimation of a low number of drag coefficients without major compromises in orbital quality. Parameterisations involving a high number of empirical parameters for the sake of deceptively small observation residuals can corrupt the geophysical significance of SLR-determined parameters.

The lower bound uncertainty of the equatorial geocentre coordinates determined from LAGEOS-Etalon data is around $1.5 \mathrm{~mm}$ and $4 \mathrm{~mm}$ for the $Z$ coordinate, higher than the target of $1 \mathrm{~mm}$ geocentre vector accuracy pursued by GGOS (Gross et al 2009). Primarily designed to test fundamental and gravitational physics, LARES may prove a useful addition to the solutions derived by the ILRS ACs for TRF determination. The modelling of non-conservative forces acting on LARES is greatly simplified by the very low area-to-mass ratio of this satellite. Our analysis shows that, on average, an improvement of $25-30 \%$ in the observability of the geocentre coordinates can be achieved by combining LAGEOS- 1 and 2 with LARES data. Future ITRF releases following ITRF2014 will directly benefit from this improvement and edge closer towards meeting the stringent origin accuracy and stability requirements imposed by highly demanding geosciences applications such as the monitoring of sea level change.

In this study, all solutions involved the estimation of OPR acceleration parameters and occasionally geopotential coefficients of degree and order 2 and higher. Both OPR parameters and geopotential coefficients are correlated with the geocentre coordinates to some extent. The annual signal of the $Z$ geocentre coordinate is likely to be absorbed by geopotential coefficients, as illustrated in Fig. 13 (right). However, we have found no conclusive evidence that OPR parameters negatively affect the observability of the geocentre coordinates when geopotential coefficients are omitted and SRP coefficients are fixed. OPR parameters are necessary to accommodate orbit modelling errors. Solutions without OPR parameters have not been generated, as such pa- 
rameterisations result in poor orbital fits and and are unlikely to be adopted by any ILRS AC.

The differences in the median formal errors reported in this study for different parameterisation are primarily driven by the strengths of statistical relationships between parameters. Collinearity diagnosis is particularly useful for identifying the effect of correlations between several parameters on the observability of the geocentre coordinates and provides optimistic formal error values that do not account for modelling errors. As argued by Kuang et al (2015), the observability of a parameter and the contamination level of its estimate by modelling errors are distinct matters since models, including surface force models, are subject to refinements. Modelling errors do not constitute an inherent limitation for the sensitivity of satellite geodetic techniques to geophysical parameters. The main factors that govern the observability are rather the intrinsic quality, the quantity and spatial distribution of observations, along with the orbital configuration. Modelling errors can, however, alias into the estimates of parameters, particularly parameters affected by collinearity issues. Strongly collinear parameters may still be satisfactorily determined if their proper signatures on the observations are considerably larger than the modelling errors.

Satellite laser ranging remains the state-of-the-art satellite geodetic technique for geocentre motion observation and is unlikely to be surpassed in the near term. Further densification of the tracking network and enhancements in data and force modelling are, however, required for the technique to achieve its potential of delivering accurate geocentre coordinates which are free of collinearity issues. Improvements in the observability of geocentre motion with SLR greatly depend on the homogenisation of the network in terms of both station distribution and tracking performance. Since this is a lengthy process with limited short-term benefits, advances in the processing of existing data sets are more likely to lead to improved geocentre motion estimates in the near future.

Acknowledgements We thank the ILRS (Pearlman et al 2002) for the continuous provision of SLR data through its two data centres and four anonymous reviewers for their constructive comments that improved the contents of this article. The Generic Mapping Tools software package (Wessel et al 2013) was used to produce Figs. 1 and 4.

\section{References}

Altamimi Z, Collilieux X, Métivier L (2011) ITRF2008: an improved solution of the international terrestrial reference frame. J Geod 85(8):457-473, doi:10.1007/s00190-011-0444-4

Angermann D, Müller H (2008) On the strength of SLR observations to realize the scale and origin of the terrestrial reference system. In: Sideris MG (ed) Observing our changing Earth, International Association of Geodesy Symposia, vol 133, Springer, pp 21-29, doi:10.1007/978-3-540-85426-5_3
Argus DF (2012) Uncertainty in the velocity between the mass center and surface of Earth. J Geophys Res 117(B10), doi:10.1029/2012JB009196

Barletta VR, Sørensen LS, Forsberg R (2013) Scatter of mass changes estimates at basin scale for Greenland and Antarctica. The Cryosphere 7(5):1411-1432, doi:10.5194/tc-7-1411-2013

Baur O, Kuhn M, Featherstone WE (2013) Continental mass change from GRACE over 2002-2011 and its impact on sea level. J Geod 87(2):117-125, doi:10.1007/s00190-012-0583-2

Blaha G (1982) Free networks: minimum norm solution as obtained by the inner adjustment constraint method. Bull Géod 56(3):209-219, doi:10.1007/BF02525582

Bouillé F, Cazenave A, Lemoine JM, Crétaux JF (2000) Geocenter motion from the DORIS space system and laser data to the Lageos satellites: comparison with surface loading data. Geophys J Int 143(1):71-82, doi:10.1046/j.1365-246x.2000.00196.x

Chambers DP (2006) Observing seasonal steric sea level variations with GRACE and satellite altimetry. J Geophys Res 111(C3), doi:10.1029/2005JC002914

Chambers DP, Wahr J, Nerem RS (2004) Preliminary observations of global ocean mass variations with GRACE. Geophys Res Lett 31(13), doi:10.1029/2004GL020461

Chen JL, Rodell M, Wilson CR, Famiglietti JS (2005) Low degree spherical harmonic influences on Gravity Recovery and Climate Experiment (GRACE) water storage estimates. Geophys Res Lett 32(14), doi:10.1029/2005GL022964

Cheng M, Tapley BD (1999) Seasonal variations in low degree zonal harmonics of the Earth's gravity field from satellite laser ranging observations. J Geophys Res 104(B2):2667-2681, doi:10.1029/1998JB900036

Cheng M, Shum CK, Tapley BD (1997) Determination of longterm changes in the Earth's gravity field from satellite laser ranging observations. J Geophys Res 102(B10):22,377-22,390, doi: $10.1029 / 97 J B 01740$

Cheng M, Ries JC, Tapley BD (2013) Geocenter variations from analysis of SLR data. In: Altamimi Z, Collilieux X (eds) Reference Frames for Applications in Geosciences, International Association of Geodesy Symposia, vol 138, Springer, pp 19-25, doi:10.1007/978-3-642-32998-2_4

Ciufolini I, Paolozzi A, Pavlis EC, Ries JC, Gurzadyan V, Koenig R, Matzner R, Penrose R, Sindoni G (2012) Testing general relativity and gravitational physics using the LARES satellite. Eur Phys J Plus 127(11):133, doi:10.1140/epjp/i2012-12133-8

Collilieux X, Wöppelmann G (2011) Global sea-level rise and its relation to the terrestrial reference frame. J Geod 85(1):9-22, doi:10.1007/s00190-010-0412-4

Collilieux X, Altamimi Z, Ray J, van Dam T, Wu X (2009) Effect of the satellite laser ranging network distribution on geocenter motion estimation. J Geophys Res 114(B4), doi:10.1029/2008JB005727

Cook GE (1965) Satellite drag coefficients. Planet Space Sci 13(10):929-946, doi:10.1016/0032-0633(65)90150-9

Crétaux JF, Soudarin L, Davidson FJM, Gennero MC, Bergé-Nguyen M, Cazenave A (2002) Seasonal and interannual geocenter motion from SLR and DORIS measurements: comparison with surface loading data. J Geophys Res 107(B12):ETG 16-1-ETG 16-9, doi:10.1029/2002JB001820

Desai SD (2002) Observing the pole tide with satellite altimetry. J Geophys Res 107(C11):7-1-7-13, doi:10.1029/2001JC001224

Dong D, Yunck T, Heflin M (2003) Origin of the International Terrestrial Reference Frame. J Geophys Res 108(B4):ETG 8-1ETG 8-10, doi:10.1029/2002JB002035

Dong D, Qu W, Fang P, Peng D (2014) Non-linearity of geocentre motion and its impact on the origin of the terrestrial reference frame. Geophys J Int 198(2):1071-1080, doi:10.1093/gji/ggu187

Feissel-Vernier M, Bail KL, Berio P, Coulot D, Ramillien G, Valette JJ (2006) Geocentre motion measured with DORIS and SLR, 
and predicted by geophysical models. J Geod 80(8-11):637-648, doi:10.1007/s00190-006-0079-z

Gobinddass ML, Willis P, Viron O, Sibthorpe A, Zelensky NP, Ries JC, Ferland R, Bar-Sever Y, Diament M (2009a) Systematic biases in DORIS-derived geocenter time series related to solar radiation pressure mis-modeling. J Geod 83(9):849-858, doi:10.1007/s00190-009-0303-8

Gobinddass ML, Willis P, Viron O, Sibthorpe A, Zelensky NP, Ries JC, Ferland R, Bar-Sever Y, Diament M, Lemoine FG (2009b) Improving DORIS geocenter time series using an empirical rescaling of solar radiation pressure models. Adv Space Res 44(11):1279-1287, doi:10.1016/j.asr.2009.08.004

Gross RS, Beutler G, Plag HP (2009) Integrated scientific and societal user requirements and functional specifications for the GGOS. In: Plag HP, Pearlman M (eds) Global Geodetic Observing System, Springer, pp 209-224, doi:10.1007/978-3-642-02687-4_7

Kuang D, Bar-Sever Y, Haines B (2015) Analysis of orbital configurations for geocenter determination with GPS and low-Earth orbiters. J Geod 89(5):471-481, doi:10.1007/s00190-015-0792-6

Lavallée DA, van Dam T, Blewitt G, Clarke PJ (2006) Geocenter motions from GPS: a unified observation model. J Geophys Res 111(B5), doi:10.1029/2005JB003784

Lejba P, Schillak S (2011) Determination of station positions and velocities from laser ranging observations to Ajisai, Starlette and Stella satellites. Adv Space Res 47(4):654-662, doi:10.1016/j.asr.2010.10.013

Lucchesi DM, Ciufolini I, Andrés JI, Pavlis EC, Peron R, Noomen R, Currie DG (2004) LAGEOS II perigee rate and eccentricity vector excitations residuals and the Yarkovsky-Schach effect. Planet Space Sci 52(8):699-710, doi:10.1016/j.pss.2004.01.007

Lyard F, Lefevre F, Letellier T, Francis O (2006) Modelling the global ocean tides: modern insights from FES2004. Ocean Dyn 56(56):394-415, doi:10.1007/s10236-006-0086-x

Maier A, Krauss S, Hausleitner W, Baur O (2012) Contribution of satellite laser ranging to combined gravity field models. Adv Space Res 49(3):556-565, doi:10.1016/j.asr.2011.10.026

Meindl M, Beutler G, Thaller D, Dach R, Jäggi A (2013) Geocenter coordinates estimated from GNSS data as viewed by perturbation theory. Adv Space Res 51(7):1047-1064, doi:10.1016/j.asr.2012.10.026

Melachroinos SA, Lemoine FG, Zelensky NP, Rowlands DD, Luthcke SB, Bordyugov O (2013) The effect of geocenter motion on Jason2 orbits and the mean sea level. Adv Space Res 51(8):1323-1334, doi:10.1016/j.asr.2012.06.004

Mendes VB, Pavlis EC (2004) High-accuracy zenith delay prediction at optical wavelengths. Geophys Res Lett 31(14), doi:10.1029/2004GL020308

Mendes VB, Prates G, Pavlis EC, Pavlis DE, Langley RB (2002) Improved mapping functions for atmospheric refraction correction in SLR. Geophys Res Lett 29(10):53-1-53-4, doi:10.1029/2001GL014394

Milani A, Nobili AM, Farinella P (1987) Non-gravitational perturbations and satellite geodesy. Adam Hilger, Bristol

Montenbruck O, Gill E (2000) Satellite orbits: models, methods and applications, 4th edn. Springer, Berlin

Montgomery DC, Peck EA, Vining GG (2012) Introduction to linear regression analysis, 5 th edn. Wiley, Hoboken, NJ

Moore P, Wang J (2003) Geocentre variation from laser tracking of LAGEOS1/2 and loading data. Adv Space Res 31(8):1927-1933, doi:10.1016/S0273-1177(03)00170-4

Moore P, Boomkamp HJ, Carnochan S, Walmsley RJ (1999) Faust: multi-satellite orbital dynamics software. Adv Space Res 23(4):785-795, doi:10.1016/S0273-1177(99)00144-1

Moore P, Zhang Q, Alothman A (2005) Annual and semiannual variations of the Earth's gravitational field from satellite laser ranging and CHAMP. J Geophys Res 110(B6), doi: $10.1029 / 2004 J B 003448$

Noll CE (2010) The crustal dynamics data information system: a resource to support scientific analysis using space geodesy. Adv Space Res 45(12):1421-1440, doi:10.1016/j.asr.2010.01.018

Otsubo T, Appleby GM (2003) System-dependent center-of-mass correction for spherical geodetic satellites. J Geophys Res 108(B4), doi:10.1029/2002JB002209

Otsubo T, Sherwood RA, Appleby GM, Neubert R (2015) Centerof-mass corrections for sub-cm-precision laser-ranging targets: Starlette, Stella and LARES. J Geod 89(4):303-312, doi:10.1007/s00190-014-0776-y

Paolozzi A, Ciufolini I (2013) LARES successfully launched in orbit: satellite and mission description. Acta Astronaut 91:313-321, doi:10.1016/j.actaastro.2013.05.011

Pavlis NK, Holmes SA, Kenyon SC, Factor JK (2012) The development and evaluation of the Earth Gravitational Model 2008 (EGM2008). J Geophys Res 117(B4), doi:10.1029/2011JB008916

Pearlman MR, Degnan JJ, Bosworth JM (2002) The International Laser Ranging Service. Adv Space Res 30(2):135-143, doi:10.1016/S0273-1177(02)00277-6

Petit G, Luzum B (2010) IERS Conventions (2010). IERS Technical Note No. 36, Verlag des Bundesamts für Kartographie und Geodäsie, Frankfurt am Main

Picone JM, Hedin AE, Drob DP, Aikin AC (2002) NRLMSISE-00 empirical model of the atmosphere: statistical comparisons and scientific issues. J Geophys Res 107(A12):SIA 15-1-SIA 15-16, doi:10.1029/2002JA009430

Rawlings JO, Pantula SG, Dickey DA (1998) Applied regression analysis: a research tool, 2nd edn. Springer, New York

Ray RD, Ponte RM (2003) Barometric tides from ECMWF operational analyses. Ann Geophys 21(8):1897-1910, doi:10.5194/angeo-211897-2003

Rebischung P, Altamimi Z, Springer T (2014) A collinearity diagnosis of the GNSS geocenter determination. J Geod 88(1):65-85, doi:10.1007/s00190-013-0669-5

Ries JC (2008) SLR center of mass offset for Starlette and Stella, URL ftp://cddis.gsfc.nasa.gov/misc/ggos/0804/ GNCWG_Ries_slrbias_080416.pdf, GGOS Working Group on Ground Networks Communications Meeting, Vienna

Rubincam DP (1988) Yarkovsky thermal drag on LAGEOS. J Geophys Res 93(B11):13,805-13,810, doi:10.1029/JB093iB11p13805

Rubincam DP (1990) Drag on the LAGEOS satellite. J Geophys Res 95(B4):4881-4886, doi:10.1029/JB095iB04p04881

Sośnica K (2014) Determination of precise satellite orbits and geodetic parameters using satellite laser ranging. PhD Dissertation, Astronomical Institute, University of Bern, Switzerland

Sośnica K, Jäggi A, Thaller D, Beutler G, Dach R (2014) Contribution of Starlette, Stella, and AJISAI to the SLR-derived global reference frame. J Geod 88(8):789-804, doi:10.1007/s00190-0140722-z

Standish EM, Newhall XX, Williams JG, Folkner WF (1995) JPL planetary and lunar ephemerides, DE403/LE403. Interoffice Memorandum 314.10-127, Jet Propulsion Laboratory, Pasadena, California

Wessel P, Smith WHF, Scharroo R, Luis J, Wobbe F (2013) Generic mapping tools: improved version released. EOS Trans Am Geophys Union 94(45):409-410, doi:10.1002/2013EO450001

Wu X, Argus DF, Heflin MB, Ivins ER, Webb FH (2002) Site distribution and aliasing effects in the inversion for load coefficients and geocenter motion from GPS data. Geophys Res Lett 29(24):63-163-4, doi:10.1029/2002GL016324

Wu X, Heflin MB, Ivins ER, Argus DF, Webb FH (2003) Large-scale global surface mass variations inferred from GPS measurements of load-induced deformation. Geophys Res Lett 30(14):SDE 5-1SDE 5-4, doi:10.1029/2003GL017546 
Wu X, Collilieux X, Altamimi Z, Vermeersen BLA, Gross RS, Fukumori I (2011) Accuracy of the International Terrestrial Reference Frame origin and Earth expansion. Geophys Res Lett 38(13), doi:10.1029/2011GL047450

Wu X, Ray J, van Dam T (2012) Geocenter motion and its geodetic and geophysical implications. J Geodyn 58:44-61, doi:10.1016/j.jog.2012.01.007

Zelensky NP, Lemoine FG, Chinn DS, Melachroinos S, Beckley BD, Beall JW, Bordyugov O (2014) Estimated SLR station position and network frame sensitivity to time-varying gravity. J Geod 88(6):517-537, doi:10.1007/s00190-014-0701-4 